\title{
HOCHSCHILD COHOMOLOGY OF ALGEBRAS OF QUATERNION TYPE, I: GENERALIZED QUATERNION GROUPS
}

\author{
A. I. GENERALOV
}

\begin{abstract}
In terms of generators and defining relations, a description is given of the Hochschild cohomology algebra for one of the series of local algebras of quaternion type. As a corollary, the Hochschild cohomology algebra is described for the group algebras of generalized quaternion groups over algebraically closed fields of characteristic 2 .
\end{abstract}

\section{INTRODUCTION}

Let $R$ be a finite-dimensional algebra over a field $K$, let $\Lambda=R^{\mathrm{e}}=R \otimes_{K} R^{\text {op }}$ be its enveloping algebra, and let $\operatorname{HH}^{n}(R)=\operatorname{Ext}_{\Lambda}^{n}(R, R)$ be the $n$th Hochschild cohomology group of the algebra $R$ (with coefficients in the $R$-bimodule $R$ ). On the vector space

$$
\mathrm{HH}^{*}(R)=\bigoplus_{n \geq 0} \mathrm{HH}^{n}(R)=\bigoplus_{n \geq 0} \operatorname{Ext}_{\Lambda}^{n}(R, R),
$$

we can introduce the $\smile$-product with respect to which it becomes an associative $K$ algebra (see [1, §5], 2. Chapter XI], 3]); this algebra is called the Hochschild cohomology algebra. The algebra $\mathrm{HH}^{*}(R)$ is a graded commutative algebra [3]; moreover, the $\smile$ product coincides with the Yoneda product on the Ext-algebra $\bigoplus_{n \geq 0} \operatorname{Ext}_{\Lambda}^{n}(R, R)$ of the $\Lambda$-module $R$ [4, p. 120].

In recent years, progress has been made in the investigation of the multiplicative structure of the Hochschild cohomology algebra for finite-dimensional algebras. In [5, 6] it was proved that $\mathrm{HH}^{*}(K[G]) \simeq \mathrm{H}^{*}(G) \otimes_{K} K[G]$ if $G$ is a commutative finite group. In [7], a description was obtained of the Hochschild cohomology algebra for the symmetric group $S_{3}$ over the field $\mathbb{F}_{3}$ and for the alternating group $A_{4}$ and the dihedral 2-groups over the field $\mathbb{F}_{2}$. In 8 , the algebra $\mathrm{HH}^{*}(R)$ was described in the case where $R$ is a self-injective Nakayama algebra, and in [9], the subalgebra $\operatorname{HH}^{r *}(R)$ generated by the homogeneous elements of degrees divisible by $r$ was identified, where $R$ is the so-called Möbius algebra (here $r$ is a parameter related to the algebra $R$ ). We note also that the additive structure of the algebra $\operatorname{HH}^{*}(R)$ was described in [10] for group blocks having tame representation type and one or three simple modules.

In the recent paper [11] by the author, a description of the Hochschild cohomology algebra was given for algebras of dihedral type in the family $D(3 \mathcal{K})$ over an algebraically closed ground field of characteristic two. We recall that algebras of dihedral, semidihedral, and quaternion types appeared in the work of Erdmann on classification of group blocks of tame representation type (see [12]). By using the results of [13, our description of the algebra $\mathrm{HH}^{*}(R)$ was extended additionally to algebras of three families in the classification of Erdmann, namely, to those of the families $D(3 \mathcal{A})_{1}, D(3 \mathcal{B})_{1}$, and $D(3 \mathcal{D})_{1}$

2000 Mathematics Subject Classification. Primary 13D03.

Key words and phrases. Algebras of quaternion type, Hochschild cohomology, generalized quaternion groups. 
(in the notation of 12]). In particular, this allowed us to give a description of the Hochschild cohomology algebra for all group blocks with dihedral defect group and three simple modules.

In the present paper, we use the technique of [11] to compute the Hochschild cohomology algebra for a family of local algebras of quaternion type, namely, for the family that contains the group algebras of generalized quaternion groups over an algebraically closed field of characteristic 2 (see [12]). The corresponding approach was used earlier in the computation of Yoneda algebras for algebras of dihedral or semidihedral type (see [14-19]). Its specific feature is that, on the basis of some empirical observations, a conjecture is stated concerning the structure of the minimal projective resolution of the modules under consideration and then, upon verifying this conjecture, the resolutions are used in the calculation of the corresponding cohomology algebras. For local algebras of quaternion type to be treated here, in the same way we build the minimal $\Lambda$-projective resolution of the module $R$ (see $\S 2$ ). With the help of this resolution, we pick a (finite) set of generators for the algebra $\mathrm{HH}^{*}(R)$ and find relations satisfied by these generators (see $\S \S 3$ and 4 ). We see that the minimal $\Lambda$-projective resolution of the bimodule $R$ has period 4 and consequently, the Hochschild cohomology of the local algebras mentioned above is also periodic. It should be noted that the initial part (containing three arrows) of the bimodule resolution was described in 20 for an arbitrary algebra. However, in the context of the family of algebras under consideration, the proof of the 4-periodicity of this resolution requires additional effort (cf. Proposition 2.5). So, it seems that our direct construction of bimodule resolutions is more efficient. We also note that a similar periodicity of bimodule resolutions is valid for the self-injective algebras of the tree class $A_{n} ;$ see [8, 9].

For completeness, in the Appendix we briefly describe the results of the calculation of the Yoneda algebra for the local algebras under consideration.

\section{§1. Statement of the Main Result}

Let $K$ be an algebraically closed field of arbitrary characteristic. For any $k \in \mathbb{N}$ with $k \geq 2$, we introduce the $K$-algebra $R_{k}=K\langle X, Y\rangle / I$, where $I$ is an ideal of the free algebra $K\langle X, Y\rangle$ generated by the elements

$$
X^{2}-Y(X Y)^{k-1}, Y^{2}-X(Y X)^{k-1},(X Y)^{k}-(Y X)^{k}, X(Y X)^{k} .
$$

The images of the elements $X$ and $Y$ under the canonical homomorphism from $K\langle X, Y\rangle$ to $R_{k}$ will be denoted by $x$ and $y$, respectively. The algebra $R_{k}$ is a symmetric local algebra of tame representation type [12, III.1]; moreover, in terms of [12, Chapter VII], $R_{k}$ is an algebra of quaternion type. If $G$ is the generalized quaternion group of order $2^{n}(n \geq 3)$ and char $K=2$, then the group algebra $K G$ is isomorphic to the algebra $R_{k}$ with $k=2^{n-2}$.

To describe the Hochschild cohomology algebra $\operatorname{HH}^{*}\left(R_{k}\right)$ for the algebras $R_{k}(k \geq 2)$, we construct several graded algebras. Set

$$
\mathcal{X}_{1}=\left\{p_{1}, p_{2}, p_{2}^{\prime}, p_{3}, u_{1}, u_{1}^{\prime}, u_{2}, v_{1}, v_{2}, v_{2}^{\prime}, w, z\right\} .
$$

The grading on the algebra $K\left[\mathcal{X}_{1}\right]$ will be such that

$$
\begin{cases}\operatorname{deg} p_{1}=\operatorname{deg} p_{2}=\operatorname{deg} p_{2}^{\prime}=\operatorname{deg} p_{3}=0, & \operatorname{deg} u_{1}=\operatorname{deg} u_{1}^{\prime}=\operatorname{deg} u_{2}=1, \\ \operatorname{deg} v_{1}=\operatorname{deg} v_{2}=\operatorname{deg} v_{2}^{\prime}=2, & \operatorname{deg} w=3, \operatorname{deg} z=4 .\end{cases}
$$


Then we introduce the graded $K$-algebra $\mathcal{A}_{1}=K\left[\mathcal{X}_{1}\right] / I_{1}$, where the ideal $I_{1}$ is generated by the following elements:

of degree 0 :

$$
\left\{\begin{array}{c}
p_{1}^{k}, p_{2}^{2},\left(p_{2}^{\prime}\right)^{2}, p_{1} p_{2}, p_{1} p_{2}^{\prime}, p_{2} p_{2}^{\prime} \\
p_{3}^{2}, p_{1} p_{3}, p_{2} p_{3}, p_{2}^{\prime} p_{3}
\end{array}\right.
$$

of degree 1:

$$
\begin{gathered}
p_{2} u_{1}-p_{2}^{\prime} u_{1}^{\prime}, p_{2}^{\prime} u_{1}-p_{1} u_{1}^{\prime}, p_{1} u_{1}-p_{2} u_{1}^{\prime} ; \\
p_{1}^{k-1} u_{2}, p_{2} u_{2}, p_{2}^{\prime} u_{2}, p_{3} u_{2}, p_{2} u_{1}-p_{1}^{k-2} u_{2} ;
\end{gathered}
$$

of degree 2 :

$$
\left\{\begin{array}{c}
p_{1}^{k-1} v_{1}, p_{2} v_{2}, p_{2}^{\prime} v_{2}^{\prime}, p_{3} v_{1}, p_{3} v_{2}, p_{3} v_{2}^{\prime} \\
p_{2} v_{1}-p_{1} v_{2}^{\prime}, p_{2} v_{1}-p_{2}^{\prime} v_{2}, p_{2} v_{1}-p_{3} u_{1}^{2} \\
p_{2}^{\prime} v_{1}-p_{1} v_{2}, p_{2}^{\prime} v_{1}-p_{2} v_{2}^{\prime}, p_{2}^{\prime} v_{1}-p_{3}\left(u_{1}^{\prime}\right)^{2} \\
u_{1} u_{1}^{\prime}-k p_{1}^{k-2} v_{1} \\
u_{2}^{2}, u_{1} u_{2}-k p_{3}\left(u_{1}^{\prime}\right)^{2}, u_{1}^{\prime} u_{2}-k p_{3} u_{1}^{2}
\end{array}\right.
$$

of degree 3 :

$$
\begin{gathered}
u_{1}^{\prime} v_{2}-u_{1} v_{2}^{\prime}, u_{1}^{\prime} v_{1}-u_{1} v_{2}, u_{1} v_{1}-u_{1}^{\prime} v_{2}^{\prime}, u_{1}^{3}-\left(u_{1}^{\prime}\right)^{3} \\
p_{2} w, p_{2}^{\prime} w, p_{3} w, u_{1}^{\prime} v_{2}-p_{1}^{k-2} w \\
u_{2} v_{2}, u_{2} v_{2}^{\prime}, u_{2} v_{1}-p_{1} w
\end{gathered}
$$

of degree 4:

$$
\begin{gathered}
v_{2}^{2},\left(v_{2}^{\prime}\right)^{2}, v_{1} v_{2}, v_{1} v_{2}^{\prime}, v_{2} v_{2}^{\prime}, v_{1}^{2}-p_{1}^{2} z ; \\
u_{1} w, u_{1}^{\prime} w, u_{2} w
\end{gathered}
$$

of degree 5 :

$$
v_{2} w, v_{2}^{\prime} w, v_{1} w+p_{1} u_{2} z
$$

of degree 6 :

$$
w^{2} .
$$

Moreover, we induce a grading on the algebra $\mathcal{A}_{1}$ from the grading on $K\left[\mathcal{X}_{1}\right]$.

Next, we consider the algebra $\mathcal{A}_{2}=K\left[\mathcal{X}_{2}\right] / I_{2}$, where

$$
\mathcal{X}_{2}=\mathcal{X}_{1} \backslash\left\{u_{2}, w\right\}
$$

with $\mathcal{X}_{1}$ as in (1.2); the grading on this algebra is introduced by restricting the grading of $K\left[\mathcal{X}_{1}\right]$ (see (1.3)), and the ideal $I_{2}$ is spanned by the generators of the ideal $I_{1}$ occurring in (1.4) with $k=2$, in (1.5) and (1.7) with $k=2$, in (1.9), and in (1.12). Since the ideal $I_{2}$ is homogeneous, the grading on the algebra $K\left\langle\mathcal{X}_{2}\right\rangle$ induces a grading on $\mathcal{A}_{2}$.

Next, consider the set

$$
\mathcal{X}_{3}=\left\{p_{1}, p_{2}, p_{2}^{\prime}, u_{2}, u_{3}, v_{0}, v_{0}^{\prime}, v_{1}, w, z\right\} .
$$

We introduce a grading on the algebra $K\left\langle\mathcal{X}_{3}\right\rangle$ so that

$$
\left\{\begin{aligned}
\operatorname{deg} p_{1}=\operatorname{deg} p_{2}=\operatorname{deg} p_{2}^{\prime}=0, & \operatorname{deg} u_{2}=\operatorname{deg} u_{3}=1, \\
\operatorname{deg} v_{0}=\operatorname{deg} v_{0}^{\prime}=\operatorname{deg} v_{1}=2, & \operatorname{deg} w=3, \operatorname{deg} z=4 .
\end{aligned}\right.
$$


Then we define a graded $K$-algebra $\mathcal{A}_{3}=K\left\langle\mathcal{X}_{3}\right\rangle / I_{3}$, where the ideal $I_{3}$ is generated by the following elements:

of degree 0 :

$$
p_{1}^{k+1}, p_{2}^{2},\left(p_{2}^{\prime}\right)^{2}, p_{1} p_{2}, p_{1} p_{2}^{\prime}, p_{2} p_{2}^{\prime}
$$

of degree 1:

$$
\begin{gathered}
p_{1}^{k-1} u_{2}, p_{2} u_{2}, p_{2}^{\prime} u_{2} ; \\
p_{1} u_{3} ;
\end{gathered}
$$

of degree 2:

$$
\begin{gathered}
p_{1} v_{0}, p_{1} v_{0}^{\prime}, p_{2} v_{0}, p_{2}^{\prime} v_{0}^{\prime}, p_{2} v_{1}, p_{2}^{\prime} v_{1}, p_{1}^{k-1} v_{1} \\
p_{2} v_{0}^{\prime}+p_{2}^{\prime} v_{0}
\end{gathered}
$$

of degree 3 :

$$
\begin{gathered}
u_{2} v_{0}, u_{2} v_{0}^{\prime} ; \\
p_{2} w, p_{2}^{\prime} w, u_{2} v_{1}-p_{1} w ; \\
u_{3} v_{1}, p_{2}^{\prime} u_{3} v_{0}-p_{1}^{k-1} w ;
\end{gathered}
$$

of degree 4:

$$
\begin{gathered}
v_{0} v_{0}^{\prime}, v_{0} v_{1}, v_{0}^{\prime} v_{1}, v_{0}^{2}-2 p_{2}^{\prime} z,\left(v_{0}^{\prime}\right)^{2}-2 p_{2} z, v_{1}^{2}+p_{1}^{2} z ; \\
u_{2} w, p_{1}^{k} z ; \\
u_{3} w ;
\end{gathered}
$$

of degree 5 :

$$
v_{0} w, v_{0}^{\prime} w, v_{1} w+p_{1} u_{2} z
$$

and, additionally, by the elements of the form

$$
a b-(-1)^{\operatorname{deg} a \operatorname{deg} b} b a \quad \text { with } a, b \in \mathcal{X}_{3} .
$$

Since the ideal $I_{3}$ is homogeneous, the grading on the algebra $K\left\langle\mathcal{X}_{3}\right\rangle$ induces a grading on $\mathcal{A}_{3}$.

Next, put

$$
\mathcal{X}_{4}=\left(\mathcal{X}_{3} \backslash\left\{u_{2}, u_{3}, w\right\}\right) \cup\left\{\widetilde{u}_{1}, \widetilde{u}_{1}^{\prime}, \widetilde{w}\right\} .
$$

A grading on the algebra $K\left\langle\mathcal{X}_{4}\right\rangle$ is introduced by the requirement that relations (1.18) be satisfied for the elements of the set $\mathcal{X}_{3} \backslash\left\{u_{2}, u_{3}, w\right\}$, and, moreover,

$$
\operatorname{deg} \widetilde{u}_{1}=\operatorname{deg} \widetilde{u}_{1}^{\prime}=1, \quad \operatorname{deg} \widetilde{w}=3 .
$$

Then we define a graded $K$-algebra $\mathcal{A}_{4}=K\left\langle\mathcal{X}_{4}\right\rangle / I_{4}$, where the ideal $I_{4}$ is generated by the generators of $I_{3}$ listed in (1.19), (1.22), and (1.27), by the elements

$$
\begin{gathered}
p_{1}^{k} \widetilde{u}_{1}, p_{1}^{k} \widetilde{u}_{1}^{\prime}, p_{2} \widetilde{u}_{1}, p_{2}^{\prime} \widetilde{u}_{1}^{\prime} ; \\
\widetilde{u}_{1} \widetilde{u}_{1}^{\prime}, p_{2}^{\prime} v_{0}+p_{2} v_{0}^{\prime}-p_{1}^{k-1} ; \\
\widetilde{u}_{1}^{\prime} v_{0}, \widetilde{u}_{1} v_{0}^{\prime}, p_{2} \widetilde{w}, p_{2}^{\prime} \widetilde{w}, \widetilde{u}_{1} v_{1}-\widetilde{u}_{1}^{\prime} v_{1}, p_{1} \widetilde{w}-2 \widetilde{u}_{1} v_{1} ; \\
\widetilde{u} 1 \widetilde{w}, \widetilde{u}_{1}^{\prime} \widetilde{w}, v_{0} \widetilde{w}, v_{0}^{\prime} \widetilde{w},
\end{gathered}
$$

and by the elements of the form

$$
a b-(-1)^{\operatorname{deg} a \operatorname{deg} b} b a \quad \text { with } a, b \in \mathcal{X}_{4} .
$$

Since the ideal $I_{4}$ is homogeneous, the grading on the algebra $K\left\langle\mathcal{X}_{4}\right\rangle$ induces a grading on $\mathcal{A}_{4}$. 
Now, we consider the set

$$
\mathcal{X}_{5}=\left\{p_{1}, p_{2}, p_{2}^{\prime}, u_{2}, u_{4}, u_{4}^{\prime}, v_{0}, v_{0}^{\prime}, v_{1}, w_{1}, w_{1}^{\prime}, w, z\right\} .
$$

We introduce the grading on the algebra $K\left\langle\mathcal{X}_{3}\right\rangle$ so that

$$
\left\{\begin{array}{l}
\operatorname{deg} p_{1}=\operatorname{deg} p_{2}=\operatorname{deg} p_{2}^{\prime}=0, \quad \operatorname{deg} u_{2}=\operatorname{deg} u_{4}=\operatorname{deg} u_{4}^{\prime}=1, \\
\operatorname{deg} v_{0}=\operatorname{deg} v_{0}^{\prime}=\operatorname{deg} v_{1}=2, \\
\operatorname{deg} z=4 .
\end{array}\right.
$$

Then we define a graded $K$-algebra $\mathcal{A}_{5}=K\left\langle\mathcal{X}_{5}\right\rangle / I_{5}$, where the ideal $I_{4}$ is generated by the generators of $I_{3}$ of the form (1.19), (1.20), (1.22), (1.24), (1.25), (1.27), (1.28), and (1.30), by the elements

$$
\left\{\begin{array}{c}
p_{1} u_{4}, p_{1} u_{4}^{\prime}, p_{2} u_{4}, p_{2} u_{4}^{\prime}, p_{2}^{\prime} u_{4}, p_{2}^{\prime} u_{4}^{\prime}, p_{2} v_{0}^{\prime}, p_{2}^{\prime} v_{0}, \\
u_{2} u_{4}, u_{2} u_{4}^{\prime}, u_{4} u_{4}^{\prime}, p_{2} w_{1}, p_{2}^{\prime} w_{1}^{\prime}, p_{1} w_{1}, p_{1} w_{1}^{\prime}, \\
u_{4} v_{0}^{\prime}, u_{4}^{\prime} v_{0}, u_{4} v_{1}, u_{1}^{\prime} v_{1}, \\
p_{2}^{\prime} w_{1}-p_{2} w_{1}^{\prime}, p_{2}^{\prime} w_{1}-u_{4} v_{0}, p_{2}^{\prime} w_{1}-u_{4}^{\prime} v_{0}^{\prime}, \\
u_{2} w_{1}, u_{2} w_{1}^{\prime}, u_{4} w_{1}, u_{4} w_{1}^{\prime}, u_{4}^{\prime} w_{1}, u_{4}^{\prime} w_{1}^{\prime}, \\
v_{0} w_{1}^{\prime}, v_{0}^{\prime} w_{1}, v_{1} w_{1}, v_{1} w_{1}^{\prime}, v_{0} w_{1}-2 u_{4} z, v_{0}^{\prime} w_{1}^{\prime}-2 u_{4}^{\prime} z, w_{1} w_{1}^{\prime}, \\
p_{2}^{\prime} w_{1}-p_{1}^{k-1} w, u_{4} w, u_{4}^{\prime} w, w_{1} w, w_{1}^{\prime} w,
\end{array}\right.
$$

and by the elements of the form

$$
a b-(-1)^{\operatorname{deg} a \operatorname{deg} b} b a \quad \text { with } a, b \in \mathcal{X}_{5} .
$$

Since the ideal $I_{5}$ is homogeneous, the grading on the algebra $K\left\langle\mathcal{X}_{5}\right\rangle$ induces a grading on $\mathcal{A}_{5}$.

Next, we consider the set

$$
\mathcal{X}_{6}=\left(\mathcal{X}_{5} \backslash\{w\}\right) \cup\{\widetilde{w}\}
$$

and introduce a grading on the algebra $K\left\langle\mathcal{X}_{6}\right\rangle$ by the requirement that relations (1.34) be satisfied for the elements of the set $\mathcal{X}_{5} \backslash\{w\}$, and, moreover,

$$
\operatorname{deg} \widetilde{w}=3 .
$$

Then we define a graded $K$-algebra $\mathcal{A}_{6}=K\left\langle\mathcal{X}_{6}\right\rangle / I_{6}$, where the ideal $I_{6}$ is generated by the generators of $I_{3}$ occurring in (1.19), (1.20), (1.22), (1.24), (1.27), and (1.35), by the elements

$$
\begin{gathered}
p_{2} \widetilde{w}, p_{2}^{\prime} \widetilde{w}, p_{2} w_{1}-\frac{1}{2} p_{1}^{k} \widetilde{w}, u_{2} v_{1}-\frac{1}{2} p_{1}^{2} \widetilde{w} \\
u_{2} \widetilde{w}, u_{4} \widetilde{w}, u_{4}^{\prime} \widetilde{w}, \widetilde{w} w_{1}, \widetilde{w} w_{1}^{\prime},
\end{gathered}
$$

and by the elements of the form

$$
a b-(-1)^{\operatorname{deg} a \operatorname{deg} b} b a \quad \text { with } a, b \in \mathcal{X}_{6} .
$$

The algebra $\mathcal{A}_{6}$ inherits the natural grading from the algebra $K\left\langle\mathcal{X}_{6}\right\rangle$.

Finally, we consider the set

$$
\mathcal{X}_{7}=\left(\mathcal{X}_{5} \backslash\left\{u_{2}, u_{4}, u_{4}^{\prime}, w_{1}, w_{1}^{\prime}, w\right\}\right) \cup\left\{\widetilde{u}_{3}\right\}
$$

and introduce a grading on the algebra $K\left\langle\mathcal{X}_{7}\right\rangle$ so that relations (1.34) be satisfied for the elements of the set $\mathcal{X}_{7} \backslash\left\{\widetilde{u}_{3}\right\}$, and, moreover,

$$
\operatorname{deg} \widetilde{u}_{3}=1 .
$$

Then we define a graded $K$-algebra $\mathcal{A}_{7}=K\left\langle\mathcal{X}_{7}\right\rangle / I_{7}$; here the ideal $I_{7}$ is generated by the generators of $I_{3}$ occurring in (1.19), (1.22), and (1.27) by the elements

$$
p_{1}^{k} \widetilde{u}_{3}, p_{2} v_{0}^{\prime}-p_{2}^{\prime} v_{0}, p_{1}^{k} z
$$


and by the elements of the form

$$
a b-(-1)^{\operatorname{deg} a \operatorname{deg} b} b a \quad \text { with } a, b \in \mathcal{X}_{7} .
$$

The algebra $\mathcal{A}_{7}$ inherits the natural grading from the algebra $K\left\langle\mathcal{X}_{7}\right\rangle$.

Theorem 1.1. Let $R=R_{k}$ where $k \in \mathbb{N} \backslash\{1\}$, and let $p=\operatorname{char} K$.

1) Assume that $p=2$.

1 a) If $k \geq 3$, then, as a graded $K$-algebra, the Hochschild cohomology algebra $\operatorname{HH}^{*}(R)$ is isomorphic to $\mathcal{A}_{1}$.

1 b) If $k=2$, then $\mathrm{HH}^{*}(R) \simeq \mathcal{A}_{2}$ as graded $K$-algebras.

2) Assume that $p=3$.

2 a) If 3 does not divide $k$, then $\mathrm{HH}^{*}(R) \simeq \mathcal{A}_{3}$ as graded $K$-algebras.

$2 \mathrm{~b}$ ) If 3 divides $k$, then $\mathrm{HH}^{*}(R) \simeq \mathcal{A}_{4}$ as graded $K$-algebras.

3) Assume that $p \notin\{2,3\}$.

3 a) If $p$ divides neither $3-2 k$, nor $k$, then $\mathrm{HH}^{*}(R) \simeq \mathcal{A}_{5}$ as graded $K$-algebras.

$3 \mathrm{~b})$ If $p$ does not divide $3-2 k$ but divides $k$, then $\mathrm{HH}^{*}(R) \simeq \mathcal{A}_{6}$ as graded $K$-algebras.

$3 \mathrm{c}$ ) If $p$ divides $3-2 k$, then $\mathrm{HH}^{*}(R) \simeq \mathcal{A}_{7}$ as graded $K$-algebras.

This description of the Hochschild cohomology algebra immediately implies the following statement.

Corollary 1.2. Let $G$ be a generalized quaternion group of order $2^{n}(n \geq 3)$, and let $K$ be an algebraically closed field of characteristic 2 . Then, with $k=2^{n-2}$, we have

$$
\mathrm{HH}^{*}(K G) \simeq \begin{cases}\mathcal{A}_{1} & \text { if } n>3, \\ \mathcal{A}_{2} & \text { if } n=3 .\end{cases}
$$

In the course of the proof of Theorem 1.1 we also compute the dimensions of the groups $\mathrm{HH}^{n}(R)$. This is of independent interest; we collect the results in the following statement.

Proposition 1.3. Let $R=R_{k}$.

1) If $p=2$, then

$$
\operatorname{dim}_{K} \mathrm{HH}^{n}(R)= \begin{cases}k+3 & \text { if } n \equiv 0,3 \quad(\bmod 4) \\ k+5 & \text { if } n \equiv 1,2 \quad(\bmod 4) .\end{cases}
$$

2) Assume that $p=3$.

2a) If 3 does not divide $k$, then

$$
\operatorname{dim}_{K} \mathrm{HH}^{n}(R)= \begin{cases}k+3 & \text { if } n=0, \\ k+2 & \text { if } n>0 .\end{cases}
$$

2b) If 3 divides $k$, then $\operatorname{dim}_{K} \mathrm{HH}^{n}(R)=k+3$ for any $n \geq 0$.

3) Assume that $p \notin\{2,3\}$.

3a) If $p$ divides neither $3-2 k$, nor $k$, then

$$
\operatorname{dim}_{K} \mathrm{HH}^{n}(R)= \begin{cases}k+3 & \text { if } n=0 \\ k+1 & \text { if } n \equiv 1,2 \quad(\bmod 4), \\ k+2 & \text { if } n>0 \text { and } n \equiv 0,3 \quad(\bmod 4) .\end{cases}
$$

3b) If $p$ does not divide $3-2 k$, but divides $k$, then

$$
\operatorname{dim}_{K} \operatorname{HH}^{n}(R)=\left\{\begin{array}{l}
k+3 \quad \text { if } n \equiv 0,3 \quad(\bmod 4), \\
k+1 \quad \text { if } n \equiv 1,2 \quad(\bmod 4) .
\end{array}\right.
$$


3c) If $p$ divides $3-2 k$, then

$$
\operatorname{dim}_{K} \mathrm{HH}^{n}(R)= \begin{cases}k+3 & \text { if } n=0, \\ k+2 & \text { if } n>0 .\end{cases}
$$

Remark 1.4. In the case where char $K=2$, Part 1 of Proposition 1.3 extends the corresponding result of [10] for group algebras of the generalized quaternion groups to the entire family of the algebras $R_{k}$.

\section{§2. Resolution}

Put $R=R_{k}(k \geq 2)$. The algebra $R$ admits the following set as a $K$-basis:

$$
\begin{aligned}
\mathcal{B}_{s t}= & \left\{(x y)^{i} \mid 0 \leq i \leq k-1\right\} \\
& \cup\left\{(y x)^{i} \mid 1 \leq i \leq k\right\} \cup\left\{x(y x)^{i}, y(x y)^{i} \mid 0 \leq i \leq k-1\right\} .
\end{aligned}
$$

This set consists of all nonzero paths of the quiver of $R$ (this quiver has one vertex and two loops $x$ and $y$ ). The set $\mathcal{B}_{s t}$ is called the standard basis of $R$. In its turn, the enveloping algebra $\Lambda$ of the algebra $R$ has a $K$-basis consisting of elements of the form

$$
u \otimes v \quad \text { with } u, v \in \mathcal{B}_{s t} .
$$

This basis of $\Lambda$ is also said to be standard. We introduce the following grading on $\Lambda$ : for an element of the form (2.1), we define its degree as the sum of the lengths of the paths $u$ and $v$, and then we put $\Lambda=\sum_{n \geq 0} \Lambda_{n}$, where $\Lambda_{n}$ is the $K$-vector space spanned by the elements (2.1) with degree $n$.

Multiplication from the right by an element $\lambda \in \Lambda$ induces an endomorphism $\lambda^{*}$ of the left $\Lambda$-module $\Lambda$; for simplicity, we often keep denoting this endomorphism by $\lambda$. Sometimes, we consider an endomorphism of the right $\Lambda$-module $\Lambda$ induced by multiplication from the left by an element $\lambda$. This endomorphism will be denoted by ${ }^{*} \lambda$.

We build the following 4 -periodic sequence in the category of $\Lambda$-modules:

$$
Q_{0} \stackrel{d_{0}}{\longleftarrow} Q_{1} \stackrel{d_{1}}{\longleftarrow} Q_{2} \stackrel{d_{2}}{\longleftarrow} Q_{3} \stackrel{d_{3}}{\longleftarrow} \cdots,
$$

where $Q_{0}=Q_{3}=\Lambda, Q_{1}=Q_{2}=\Lambda^{2}$,

$$
d_{0}=(x \otimes 1-1 \otimes x, \quad y \otimes 1-1 \otimes y),
$$

$$
\begin{aligned}
& d_{1}=\left(\begin{array}{r}
x \otimes 1+1 \otimes x-\sum_{i=0}^{k-2}(y x)^{i} y \otimes y(x y)^{k-2-i}, \quad-\sum_{i=0}^{k-1}(x y)^{i} \otimes(y x)^{k-1-i} \\
-\sum_{i=0}^{k-1}(y x)^{i} \otimes(x y)^{k-1-i}, \quad y \otimes 1+1 \otimes y-\sum_{i=0}^{k-2}(x y)^{i} x \otimes x(y x)^{k-2-i}
\end{array}\right), \\
& d_{2}=\left(\begin{array}{l}
x \otimes 1-1 \otimes x \\
y \otimes 1-1 \otimes y
\end{array}\right), \\
& d_{3}=g^{*} \quad \text { with } g=(x \otimes 1+1 \otimes x) \cdot \sum_{i=0}^{k-1}(y x)^{i} \otimes(x y)^{k-1-i} \cdot(y \otimes 1+1 \otimes y),
\end{aligned}
$$

and, for $0 \leq j \leq 3$ and $l \in \mathbb{N}$,

$$
Q_{4 l+j}=Q_{j}, \quad d_{4 l+j}=d_{j} .
$$

Also, we consider the homomorphism $\mu: Q_{0}=\Lambda \rightarrow R$ induced by the product in $R$ : $\mu(a \otimes b)=a b$. 
Theorem 2.1. The sequence (2.2) together with the augmentation map $\mu$ is the minimal $\Lambda$-projective resolution of the module $R$; in particular, the $\Lambda$-module $R$ is $\Omega$-periodic with period 4 , i.e., $\Omega^{4}\left({ }_{\Lambda} R\right) \simeq{ }_{\Lambda} R$.

Remark 2.2. With the help of Happel's lemma 22] (see also a refinement in 23]), the description of the modules $Q_{j}$ in the resolution (2.2) could be deduced from the construction of the minimal projective resolution of a unique simple $R$-module that is given in the Appendix below. However, we obtain this description directly in the course of the proof of Theorem 2.1 .

Remark 2.3. The fact that (2.2) is a differential sequence is established by direct calculations. In order to verify the formula $d_{2} d_{3}=0=d_{3} d_{4}$, we may use the relation

$$
g=(y \otimes 1+1 \otimes y) \cdot \sum_{i=0}^{k-1}(x y)^{i} \otimes(y x)^{k-1-i} \cdot(x \otimes 1+1 \otimes x) .
$$

The exactness at the member $Q_{0}$, i.e., the relation $\operatorname{Ker} \mu=\operatorname{Im} d_{0}$, is a well-known fact (see, e.g., [21, Proposition 2.1]).

We establish the exactness of the sequence at $Q_{1}$ by proving the following lemma.

Lemma 2.4. $\operatorname{Ker} d_{0} \subset \operatorname{Im} d_{1}$.

Proof. Let $s=(q, \widetilde{q}) \in \operatorname{Ker} d_{0} \subset Q_{1}=\Lambda^{2}$, i.e.,

$$
q \cdot(x \otimes 1-1 \otimes x)+\widetilde{q} \cdot(y \otimes 1-1 \otimes y)=0 .
$$

Let $q^{(t)}$ and $\widetilde{q}^{(t)}$ be the homogeneous components of degree $t(0 \leq t \leq 4 k)$ of $q$ and $\widetilde{q}$, respectively. From (2.5) it easily follows that $q^{(0)}=0=\widetilde{q}^{(0)}$. We fix $t$ such that the homogeneous components of $q$ and $\widetilde{q}$ of degree $i$ with $i<t$ are equal to zero. We shall modify $s$ successively, by adding elements of $\operatorname{Im} d_{1}$, so as to get an element all homogeneous components of which of degrees $i \leq t$ are zero. After finitely many steps, we replace the initial $s \in \operatorname{Ker} d_{0}$ by the zero element; this will yield the inclusion Ker $d_{0} \subset$ $\operatorname{Im} d_{1}$.

We introduce additional notation, letting $h_{1}$ (respectively, $h_{2}$ ) denote the first (respectively, second) column of the matrix of the differential $d_{1}$, which is viewed as an element in $\operatorname{Im} d_{1} \subset Q_{1}$.

Step 1. First, we consider the case where $t=2 \ell+1$ with $0 \leq \ell \leq k-2$. We represent the homogeneous components $q^{(t)}$ and $\widetilde{q}^{(t)}$ as linear combinations of the standard basis elements in $\Lambda$ (with coefficients $\alpha_{i}, \ldots, f_{i}, \widetilde{\alpha}_{i}, \ldots, \widetilde{f}_{i}$ in $K$ ):

$$
\begin{aligned}
q^{(t)}= & \sum_{i=0}^{\ell} \alpha_{i}(x y)^{i} \otimes x(y x)^{\ell-i}+\sum_{i=0}^{\ell} \beta_{i}(x y)^{i} \otimes y(x y)^{\ell-i} \\
& +\sum_{i=1}^{\ell} \gamma_{i}(y x)^{i} \otimes x(y x)^{\ell-i}+\sum_{i=1}^{\ell} \varphi_{i}(y x)^{i} \otimes y(x y)^{\ell-i} \\
& +\sum_{i=0}^{\ell} a_{i} x(y x)^{i} \otimes(x y)^{\ell-i}+\sum_{i=0}^{\ell-1} b_{i} x(y x)^{i} \otimes(y x)^{\ell-i} \\
& +\sum_{i=0}^{\ell} c_{i} y(x y)^{i} \otimes(x y)^{\ell-i}+\sum_{i=1}^{\ell-1} f_{i} y(x y)^{i} \otimes(y x)^{\ell-i},
\end{aligned}
$$




$$
\begin{aligned}
\widetilde{q}^{(t)}= & \sum_{i=0}^{\ell} \widetilde{\alpha}_{i}(y x)^{i} \otimes y(x y)^{\ell-i}+\sum_{i=0}^{\ell} \widetilde{\beta}_{i}(y x)^{i} \otimes x(y x)^{\ell-i} \\
& +\sum_{i=1}^{\ell} \widetilde{\gamma}_{i}(x y)^{i} \otimes y(x y)^{\ell-i}+\sum_{i=1}^{\ell} \widetilde{\varphi}_{i}(x y)^{i} \otimes x(y x)^{\ell-i} \\
& +\sum_{i=0}^{\ell} \widetilde{a}_{i} y(x y)^{i} \otimes(y x)^{\ell-i}+\sum_{i=0}^{\ell-1} \widetilde{b}_{i} y(x y)^{i} \otimes(x y)^{\ell-i} \\
& +\sum_{i=0}^{\ell} \widetilde{c}_{i} x(y x)^{i} \otimes(y x)^{\ell-i}+\sum_{i=1}^{\ell-1} \widetilde{f}_{i} x(y x)^{i} \otimes(x y)^{\ell-i} .
\end{aligned}
$$

It should be noted that the form of $\widetilde{q}^{(t)}$ corresponds to the form of $q^{(t)}$ via the obvious symmetry $x \mapsto y, y \mapsto x$. Substituting (2.6) and (2.7) in (2.5), we obtain the following equations for the scalar coefficients in (2.6) and (2.7) (for the reader's convenience, in brackets we indicate the elements of the standard basis of $R$ at which the corresponding scalars are compared):

$$
\begin{array}{lll}
\alpha_{i}=b_{i} \quad \text { for } 0 \leq i \leq \ell-1 & & \left((x y)^{i} x \otimes(x y)^{\ell-i} x\right) ; \\
\alpha_{\ell}=a_{\ell} & & \left((x y)^{\ell} x \otimes x\right) ; \\
\beta_{i}=\widetilde{f}_{i} \quad \text { for } 0 \leq i \leq \ell-1 & & \left((x y)^{i} x \otimes(y x)^{\ell-i} y\right) ; \\
\beta_{i}=\widetilde{f}_{i-1} \quad \text { for } 1 \leq i \leq \ell & & \left((x y)^{i} \otimes(x y)^{\ell+1-i}\right) ; \\
\varphi_{i}=c_{i-1} \quad \text { for } 1 \leq i \leq \ell & & \left((y x)^{i} \otimes(x y)^{l+1-i}\right) ; \\
c_{\ell}=\widetilde{\beta}_{\ell} & & \left((y x)^{\ell} y \otimes x\right) ; \\
c_{\ell}=0 & & \left((y x)^{\ell+1} \otimes 1\right) ; \\
\beta_{0}=0 & & \left(1 \otimes(x y)^{\ell+1}\right) .
\end{array}
$$

By the symmetry mentioned above, we also obtain the following equations (here we do not indicate the corresponding basis vectors):

$$
\begin{gathered}
\widetilde{\alpha}_{i}=\widetilde{b}_{i}(0 \leq i \leq \ell-1) ; \quad \widetilde{\alpha}_{\ell}=\widetilde{a}_{\ell} ; \quad \widetilde{\beta}_{i}=f_{i}(0 \leq i \leq \ell-1) ; \\
\widetilde{\beta}_{i}=f_{i-1}(1 \leq i \leq \ell) ; \quad \widetilde{\varphi}_{i}=\widetilde{c}_{i-1}(1 \leq i \leq \ell) ; \quad \widetilde{c}_{\ell}=\beta_{\ell}=0 ; \quad \widetilde{\beta}_{0}=0 .
\end{gathered}
$$

Relations (2.10), (2.11), and (2.13) imply that

$$
0=\beta_{0}=\widetilde{f}_{0}=\beta_{1}=\widetilde{f}_{1}=\beta_{2}=\cdots=\widetilde{f}_{\ell-1}=\beta_{\ell} .
$$

By (2.8), for $0 \leq i \leq \ell-1$ we have

$$
\alpha_{i}(x y)^{i} \otimes x(y x)^{\ell-i}+b_{i} x(y x)^{i} \otimes(y x)^{\ell-i}=\alpha_{i}(x y)^{i} \otimes(y x)^{\ell-i} \cdot(1 \otimes x+x \otimes 1) .
$$

Hence, replacing $s$ with

$$
s^{\prime}=\left(q^{\prime}, \widetilde{q}^{\prime}\right):=s-\sum_{i=0}^{\ell-1} \alpha_{i}(x y)^{i} \otimes(y x)^{\ell-i} \cdot h_{1},
$$


we get an element for which the corresponding homogeneous component $q^{\prime(t)}$ in $q^{\prime}$ of degree $t$ involves no nonzero summands of the form

$$
\sum_{i=0}^{\ell-1} \alpha_{i}^{\prime}(x y)^{i} \otimes x(y x)^{\ell-i}+\sum_{i=0}^{\ell-1} b_{i}^{\prime} x(y x)^{i} \otimes(y x)^{\ell-i}
$$

with $\alpha_{i}^{\prime}, b_{i}^{\prime} \in K$. Moreover, under such a replacement we can change only homogeneous components of $q$ and $\widetilde{q}$ with degrees exceeding $t$. Hence, we may assume that, already for the initial element $s$, we have $\alpha_{i}=0=b_{i}$ with $0 \leq i \leq \ell-1$.

Next, we have

$$
\begin{aligned}
\varphi_{i}(y x)^{i} \otimes y(x y)^{\ell-i}+c_{i-1} y(x y)^{i-1} \otimes(x y)^{\ell+1-i} \\
=\varphi_{i} y(x y)^{i-1} \otimes y(x y)^{\ell-i} \cdot(x \otimes 1+1 \otimes x), \\
\gamma_{i}(y x)^{i} \otimes x(y x)^{\ell-i} \\
\quad=\gamma_{i}(y x)^{i} \otimes(y x)^{\ell-i} \cdot(x \otimes 1+1 \otimes x)
\end{aligned}
$$

for $1 \leq i \leq \ell$,

$$
a_{i} x(y x)^{i} \otimes(x y)^{\ell-i}=a_{i}(x y)^{i} \otimes(x y)^{\ell-i} \cdot(x \otimes 1+1 \otimes x)
$$

for $0 \leq i \leq \ell-1$, and

$$
\alpha_{\ell}(x y)^{\ell} \otimes x+a_{\ell} x(y x)^{\ell} \otimes 1=\alpha_{\ell}(x y)^{\ell} \otimes 1 \cdot(x \otimes 1+1 \otimes x) .
$$

As before (cf. (2.15) ), we modify $s$ with the help of a suitable multiple of the column $h_{1}$, obtaining a new element such that all coefficients in the decomposition of its component of degree $t$ (this decomposition is similar to (2.6) ) are zero. Hence, we may assume that for the initial $s$ we have $q^{(t)}=0$. By symmetry, we may also assume that $\widetilde{q}^{(t)}=0$ (to establish this, we can argue as above, using the column $h_{2}$ in place of $h_{1}$ ).

Step 2 . Now we put $t=2 \ell$ with $1 \leq \ell \leq k-1$. Then

$$
\begin{aligned}
q^{(t)}= & \sum_{i=0}^{\ell} \alpha_{i}(x y)^{i} \otimes(x y)^{\ell-i}+\sum_{i=0}^{\ell-1} \beta_{i}(x y)^{i} \otimes(y x)^{\ell-i} \\
& +\sum_{i=1}^{\ell} \gamma_{i}(y x)^{i} \otimes(x y)^{\ell-i}+\sum_{i=1}^{\ell-1} \varphi_{i}(y x)^{i} \otimes(y x)^{\ell-i} \\
& +\sum_{i=0}^{\ell-1} a_{i} x(y x)^{i} \otimes x(y x)^{\ell-1-i}+\sum_{i=0}^{\ell-1} b_{i} x(y x)^{i} \otimes y(x y)^{\ell-1-i} \\
& +\sum_{i=0}^{\ell-1} c_{i} y(x y)^{i} \otimes x(y x)^{\ell-1-i}+\sum_{i=0}^{\ell-1} f_{i} y(x y)^{i} \otimes y(x y)^{\ell-1-i}, \\
\widetilde{q}^{(t)}= & \sum_{i=0}^{\ell} \widetilde{\alpha}_{i}(y x)^{i} \otimes(y x)^{\ell-i}+\sum_{i=0}^{\ell-1} \widetilde{\beta}_{i}(y x)^{i} \otimes(x y)^{\ell-i} \\
& +\sum_{i=1}^{\ell} \widetilde{\gamma}_{i}(x y)^{i} \otimes(y x)^{\ell-i}+\sum_{i=1}^{\ell-1} \widetilde{\varphi}_{i}(x y)^{i} \otimes(x y)^{\ell-i} \\
& +\sum_{i=0}^{\ell-1} \widetilde{a}_{i} y(x y)^{i} \otimes y(x y)^{\ell-1-i}+\sum_{i=0}^{\ell-1} \widetilde{b}_{i} y(x y)^{i} \otimes x(y x)^{\ell-1-i} \\
& +\sum_{i=0}^{\ell-1} \widetilde{c}_{i} x(y x)^{i} \otimes y(x y)^{\ell-1-i}+\sum_{i=0}^{\ell-1} \widetilde{f}_{i} x(y x)^{i} \otimes x(y x)^{\ell-1-i},
\end{aligned}
$$


where $\alpha_{i}, \ldots, f_{i}, \widetilde{\alpha}_{i}, \ldots, \widetilde{f}_{i} \in K$. As at Step 1, the notation in (2.20) and (2.21) employs the symmetry mentioned above. Substituting (2.20) and (2.21) in (2.5), we obtain the following equations (again we indicate the corresponding basis elements):

$$
\begin{aligned}
& \alpha_{i}=b_{i} \quad \text { for } \quad 0 \leq i \leq \ell-1 \quad\left((x y)^{i} x \otimes(x y)^{\ell-i}\right) \text {; } \\
& \alpha_{\ell}=\widetilde{f}_{\ell-1} \quad\left((x y)^{\ell} \otimes x\right) ; \\
& \beta_{i}=\tilde{f}_{i} \quad \text { for } \quad 0 \leq i \leq \ell-1 \quad\left((x y)^{i} x \otimes(y x)^{\ell-i}\right) \text {; } \\
& \beta_{i}=\widetilde{f}_{i-1} \quad \text { for } \quad 1 \leq i \leq \ell-1 \quad\left((x y)^{i} \otimes(x y)^{\ell-i} x\right) \text {; } \\
& \varphi_{i}=c_{i-1} \quad \text { for } \quad 1 \leq i \leq \ell-1 \quad\left((y x)^{i} \otimes(x y)^{\ell-i} x\right) \text {; } \\
& \gamma_{\ell}=c_{\ell-1} \quad\left((y x)^{\ell} \otimes x\right) ; \\
& \beta_{0}=0 \quad\left(1 \otimes(x y)^{\ell} x\right) ; \\
& \alpha_{\ell}=0 \quad\left((x y)^{\ell} x \otimes 1\right) .
\end{aligned}
$$

Relations (2.22)-(2.24) imply

$$
0=\beta_{0}=\widetilde{f}_{0}=\beta_{1}=\widetilde{f}_{1}=\cdots=\widetilde{f}_{\ell-2}=\beta_{\ell-1}=\widetilde{f}_{\ell-1} .
$$

Since

$$
\alpha_{i}(x y)^{i} \otimes(y x)^{\ell-i}+b_{i} x(y x)^{i} \otimes y(x y)^{\ell-1-i}=\alpha_{i}(x y)^{i} \otimes y(x y)^{\ell-1-i} \cdot(1 \otimes x+x \otimes 1)
$$

for $0 \leq i \leq \ell-1$, we have

$$
\begin{gathered}
\varphi_{i}(y x)^{i} \otimes(y x)^{\ell-i}+c_{i-1} y(x y)^{i-1} \otimes x(y x)^{\ell-i}=\varphi_{i} y(x y)^{i-1} \otimes(y x)^{\ell-i} \cdot(x \otimes 1+1 \otimes x), \\
\gamma_{i}(y x)^{i} \otimes(x y)^{\ell-i}=\gamma_{i}(y x)^{i-1} y \otimes(x y)^{\ell-i} \cdot(x \otimes 1+1 \otimes x)
\end{gathered}
$$

for $1 \leq i \leq \ell-1$, and

$$
\begin{aligned}
\gamma_{\ell}(y x)^{\ell} \otimes 1+c_{\ell-1} y(x y)^{\ell-1} \otimes x & =\gamma_{\ell} y(x y)^{\ell-1} \otimes 1 \cdot(x \otimes 1+1 \otimes x), \\
a_{i} x(y x)^{i} \otimes x(y x)^{\ell-1-i} & =a_{i}(x y)^{i} \otimes x(y x)^{\ell-1-i} \cdot(x \otimes 1+1 \otimes x)
\end{aligned}
$$

for $0 \leq i \leq \ell-2$. Moreover, for $\ell>1$ we have

$$
a_{\ell-1} x(y x)^{\ell-1} \otimes x=a_{\ell-1}(x y)^{\ell-1} \otimes 1 \cdot(x \otimes 1+1 \otimes x),
$$

and if $\ell=1$, then

$$
a_{0} x \otimes x=a_{0} x \otimes 1 \cdot\left(x \otimes 1+1 \otimes x-y(x y)^{k-1} \otimes 1\right) .
$$

Now, changing $s$ with the help of a suitable multiple of the column $h_{1}$ (cf. Step 1), we may assume that $q^{(t)}=0$ for the initial $s$; by symmetry, we may also assume that $\widetilde{q}^{(t)}=0$.

Step 3. Let $t=2 k-1$. Again we represent the homogeneous components $q^{(t)}$ and $\widetilde{q}^{(t)}$ in the form (2.6) and (2.7), respectively; here we put $\ell=k-1$. Substituting this in (2.5) and arguing as at Step 1, we obtain equations (2.8) (2.12) (again, $\ell=k-1$ ) and the equations

$$
\begin{aligned}
c_{k-1}+\widetilde{c}_{k-1} & =0 ; \\
c_{k-1} & =\widetilde{\beta}_{k-1} ; \\
\beta_{0}+\widetilde{\beta}_{0} & =0 .
\end{aligned}
$$


Relations (2.10) and (2.11) imply

$$
\beta:=\beta_{0}=\widetilde{f}_{0}=\beta_{1}=\widetilde{f}_{1}=\cdots=\widetilde{f}_{k-2}=\beta_{k-1} .
$$

Using symmetry and also (2.25) and (2.26), we obtain

$$
\widetilde{\beta}_{0}=f_{0}=\widetilde{\beta}_{1}=f_{1}=\cdots=f_{k-2}=\widetilde{\beta}_{k-1}=c_{k-1}=-\beta .
$$

Arguing as at Step 1 (see (2.14) and (2.16) $-(2.19)$ ), we may assume that for $q^{(t)}$,

$$
\begin{array}{lll}
\alpha_{i}=0(0 \leq i \leq k-1) ; & b_{i}=0(0 \leq i \leq k-2) ; & \gamma_{i}=0(1 \leq i \leq k-1) ; \\
a_{i}=0(0 \leq i \leq k-1) ; & \varphi_{i}=0(1 \leq i \leq k-1) ; & c_{i}=0(0 \leq i \leq k-2) .
\end{array}
$$

Symmetry allows us to assume that for the homogeneous component $\widetilde{q}^{(t)}$ the corresponding scalars are also zero (i.e., in the above formulas we can insert the tilde above the scalars). Thus,

$$
u:=\left(\begin{array}{c}
q^{(t)} \\
\widetilde{q}^{(t)}
\end{array}\right)=\beta \cdot\left(\begin{array}{c}
\sum_{i=0}^{k-1}(x y)^{i} \otimes y(x y)^{k-1-i}-\sum_{i=0}^{k-1} y(x y)^{i} \otimes(y x)^{k-1-i} \\
-\sum_{i=0}^{k-1}(y x)^{i} \otimes x(y x)^{k-1-i}+\sum_{i=0}^{k-1} x(y x)^{i} \otimes(x y)^{k-1-i}
\end{array}\right) .
$$

A direct calculation shows that

$$
u=\beta \cdot(1 \otimes x-x \otimes 1) \cdot h_{1} \in \operatorname{Im} d_{1} .
$$

Hence, replacing $s$ by $s-u$, we may assume that $q^{(t)}=0=\widetilde{q}^{(t)}$ for the initial $s$.

Step 4 . Let $t=2 \ell$ with $k \leq \ell \leq 2 k-1$, and put $\bar{\ell}=\ell-k$. The decomposition of the homogeneous components $q^{(t)}$ and $\widetilde{q}^{(t)}$ in the standard basis can be written as follows:

$$
\begin{aligned}
q^{(t)}= & \sum_{i=\bar{\ell}}^{k} \alpha_{i}(x y)^{i} \otimes(x y)^{\ell-i}+\sum_{i=\bar{\ell}+1}^{k} \beta_{i}(x y)^{i} \otimes(y x)^{\ell-i} \\
& +\sum_{i=\bar{\ell}}^{k-1} \gamma_{i}(y x)^{i} \otimes(x y)^{\ell-i}+\sum_{i=\bar{\ell}+1}^{k-1} \varphi_{i}(y x)^{i} \otimes(y x)^{\ell-i} \\
& +\sum_{i=\bar{\ell}}^{k-1} a_{i} x(y x)^{i} \otimes x(y x)^{\ell-1-i}+\sum_{i=\bar{\ell}}^{k-1} b_{i} x(y x)^{i} \otimes y(x y)^{\ell-1-i} \\
& +\sum_{i=\bar{\ell}}^{k-1} c_{i} y(x y)^{i} \otimes x(y x)^{\ell-1-i}+\sum_{i=\bar{\ell}}^{k-1} f_{i} y(x y)^{i} \otimes y(x y)^{\ell-1-i}, \\
\widetilde{q}^{(t)}= & \sum_{i=\bar{\ell}}^{k} \widetilde{\alpha}_{i}(y x)^{i} \otimes(y x)^{\ell-i}+\sum_{i=\bar{\ell}+1}^{k} \widetilde{\beta}_{i}(y x)^{i} \otimes(x y)^{\ell-i} \\
& +\sum_{i=\bar{\ell}}^{k-1} \widetilde{\gamma}_{i}(x y)^{i} \otimes(y x)^{\ell-i}+\sum_{i=\bar{\ell}+1}^{k-1} \widetilde{\varphi}_{i}(x y)^{i} \otimes(x y)^{\ell-i} \\
& +\sum_{i=\bar{\ell}}^{k-1} \widetilde{a}_{i} y(x y)^{i} \otimes y(x y)^{\ell-1-i}+\sum_{i=\bar{\ell}}^{k-1} \widetilde{b}_{i} y(x y)^{i} \otimes x(y x)^{\ell-1-i} \\
& +\sum_{i=\bar{\ell}}^{k-1} \widetilde{c}_{i} x(y x)^{i} \otimes y(x y)^{\ell-1-i}+\sum_{i=\bar{\ell}}^{k-1} \widetilde{f}_{i} x(y x)^{i} \otimes x(y x)^{\ell-1-i},
\end{aligned}
$$

where $\alpha_{i}, \ldots, f_{i}, \widetilde{\alpha}_{i}, \ldots, \widetilde{f}_{i} \in K$. In fact, the case where $\ell=k$ differs a little from the other cases treated at this step. We include this case in the arguments below, assuming that, for $\ell=k$, in (2.27) and (2.28) we have $\alpha_{k}=0=\widetilde{\alpha}_{k}$. 
Substituting (2.27) and (2.28) in (2.5), we obtain

$$
\begin{aligned}
& \alpha_{i}=b_{i} \text { and } \varphi_{i}=c_{i-1} \text { for } \bar{\ell}+1 \leq i \leq k-1 ; \\
& \beta_{i}=\widetilde{f}_{i} \text { and } \beta_{i}=\widetilde{f}_{i-1} \text { for } \bar{\ell}+1 \leq i \leq k-1 ; \\
& \beta_{k}-c_{k-1}-\widetilde{f}_{k-1}=0 ; \\
& \alpha_{\bar{\ell}}-b_{\bar{\ell}}-\widetilde{f}_{\bar{\ell}}=0 .
\end{aligned}
$$

As at Step 2, relations (2.29) allow us to assume that, for the initial element $s \in \operatorname{Ker} d_{0}$, we have

$$
\begin{aligned}
\alpha_{i}=b_{i}=\varphi_{i}=0 & \text { if } \quad \bar{\ell}+1 \leq i \leq k-1 \\
c_{i}=0 & \text { if } \quad \bar{\ell} \leq i \leq k-2 ; \\
a_{i}=0 & \text { if } \quad \bar{\ell} \leq i \leq k-1 .
\end{aligned}
$$

By symmetry, we may assume that for the homogeneous component $\widetilde{q}^{(t)}$ the corresponding coefficients are also zero (i.e., in the above formulas we can insert the tilde above the coefficients). Moreover, relation (2.30) implies

$$
\beta:=\widetilde{f}_{\bar{\ell}}=\beta_{\bar{\ell}+1}=\widetilde{f}_{\bar{\ell}+1}=\cdots=\beta_{k-1}=\widetilde{f}_{k-1} .
$$

By symmetry, we also have

$$
\widetilde{\beta}:=f_{\bar{\ell}}=\widetilde{\beta}_{\bar{\ell}+1}=f_{\bar{\ell}+1}=\cdots=\widetilde{\beta}_{k-1}=f_{k-1} .
$$

Next, replacing $s$ by the element

$$
s-\left(c_{k-1} y(x y)^{k-1} \otimes(y x)^{\bar{\ell}}+b_{\bar{\ell}}(x y)^{\bar{\ell}} \otimes y(x y)^{k-1}\right) \cdot h_{1},
$$

we may assume that $c_{k-1}=0=b_{\bar{\ell}}$ for the initial $s$. Then relations (2.31) and (2.32) imply that $\alpha_{\bar{\ell}}=\beta=\beta_{k}$. Similarly, we may assume that $\widetilde{\alpha}_{\bar{\ell}}=\widetilde{\beta}=\widetilde{\beta}_{k}$. Thus,

$$
\left(\begin{array}{c}
q^{(t)} \\
\widetilde{q}^{(t)}
\end{array}\right)=\left(\begin{array}{l}
\beta \cdot \sum_{i=\bar{\ell}}^{k}(x y)^{i} \otimes(y x)^{\ell-i}+\widetilde{\beta} \cdot \sum_{i=\bar{\ell}}^{k-1} y(x y)^{i} \otimes y(x y)^{\ell-1-i} \\
\widetilde{\beta} \cdot \sum_{i=\bar{\ell}}^{k}(y x)^{i} \otimes(x y)^{\ell-i}+\beta \cdot \sum_{i=\bar{\ell}}^{k-1} x(y x)^{i} \otimes x(y x)^{\ell-1-i}
\end{array}\right) .
$$

If $\ell>k$, then, replacing $s$ with the element

$$
s+\beta\left(x \otimes x(y x)^{\bar{\ell}}-y(x y)^{k-1} \otimes(y x)^{\bar{\ell}}\right) \cdot h_{1},
$$

we may assume that $\beta=0$. For $\ell=k$, the replacement of $s$ with

$$
s+\beta\left(x \otimes x-x^{2} \otimes 1-1 \otimes x^{2}\right) \cdot h_{1}
$$

again allows us to assume that $\beta=0$. Arguing by symmetry (with the use of the column $h_{2}$ ) we may assume that for the initial $s$ we also have $\widetilde{\beta}=0$. 
Step 5. Consider the case where $t=2 \ell+1$ with $k \leq \ell \leq 4 k-2$. The homogeneous component $q^{(t)}$ is represented in the form

$$
\begin{aligned}
q^{(t)}= & \sum_{i=\bar{\ell}+1}^{k} \alpha_{i}(x y)^{i} \otimes x(y x)^{\ell-i}+\sum_{i=\bar{\ell}+1}^{k} \beta_{i}(x y)^{i} \otimes y(x y)^{\ell-i} \\
& +\sum_{i=\bar{\ell}+1}^{k-1} \gamma_{i}(y x)^{i} \otimes x(y x)^{\ell-i}+\sum_{i=\bar{\ell}+1}^{k-1} \varphi_{i}(y x)^{i} \otimes y(x y)^{\ell-i} \\
& +\sum_{i=\bar{\ell}}^{k-1} a_{i} x(y x)^{i} \otimes(x y)^{\ell-i}+\sum_{i=\bar{\ell}+1}^{k-1} b_{i} x(y x)^{i} \otimes(y x)^{\ell-i} \\
& +\sum_{i=\bar{\ell}}^{k-1} c_{i} y(x y)^{i} \otimes(x y)^{\ell-i}+\sum_{i=\bar{\ell}+1}^{k-1} f_{i} y(x y)^{i} \otimes(y x)^{\ell-i} .
\end{aligned}
$$

The homogeneous component $\widetilde{q}^{(t)}$ has a similar representation, obtained by using the symmetry mentioned above (the corresponding coefficients can be supplied with the tilde; cf. (2.6) and (2.7)). Then equation (2.5) implies the relations

$$
\begin{aligned}
\alpha_{i}=b_{i} \quad \text { for } \quad \bar{\ell}+1 \leq i \leq k-1 ; \quad \varphi_{i} & =c_{i-1} \quad \text { for } \quad \bar{\ell}+2 \leq i \leq k-1 ; \\
\beta_{i}=\widetilde{f}_{i} \quad \text { for } \quad \bar{\ell}+1 \leq i \leq k-1 ; \quad \beta_{i} & =\widetilde{f}_{i-1} \quad \text { for } \quad \bar{\ell}+2 \leq i \leq k-1 ; \\
& \beta_{k}-c_{k-1}-\widetilde{f}_{k-1}=0 ; \\
\beta_{\bar{\ell}+1}+\widetilde{\varphi}_{\bar{\ell}+1}-\widetilde{c}_{\bar{\ell}} & =0 ; \\
\widetilde{\beta}_{\bar{\ell}+1}+\varphi_{\bar{\ell}+1}-c_{\bar{\ell}} & =0 .
\end{aligned}
$$

From (2.34) it follows that

$$
\beta:=\beta_{\bar{\ell}+1}=\widetilde{f}_{\bar{\ell}+1}=\cdots=\widetilde{f}_{k-2}=\beta_{k-1}=\widetilde{f}_{k-1} .
$$

By symmetry, we obtain

$$
\widetilde{\beta}:=\widetilde{\beta}_{\bar{\ell}+1}=f_{\bar{\ell}+1}=\cdots=f_{k-2}=\widetilde{\beta}_{k-1}=f_{k-1} .
$$

As at Step 1, relations (2.33) allow us to assume that for $s$ we have

$$
\begin{array}{llll}
\alpha_{i}=b_{i}=\gamma_{i}=0 & \text { for } \quad \bar{\ell}+1 \leq i \leq k-1 ; & a_{i}=0 & \text { for } \quad \bar{\ell} \leq i \leq k-1 ; \\
\varphi_{i}=0 & \text { for } \quad \bar{\ell}+2 \leq i \leq k-1 ; & c_{i}=0 & \text { for } \quad \bar{\ell}+1 \leq i \leq k-2 .
\end{array}
$$

Moreover, replacing $s$ with

$$
s-\alpha_{k}(x y)^{k} \otimes(y x)^{\bar{\ell}} \cdot h_{1},
$$

we assume also that $\alpha_{k}=0$. Observe that we may assume additionally that $c_{k-1}=0$. Indeed, the replacement of $s=(q, \widetilde{q})$ with the element

$$
s-c_{k-1} y(x y)^{k-1} \otimes y(x y)^{\bar{\ell}} \cdot h_{1}
$$

affects only $\beta_{k}$ (besides $c_{k-1}$ ) and several homogeneous components of $q$ and $\widetilde{q}$ of degree greater than $t$. Similarly, replacing $s$ with

$$
s-\varphi_{\bar{\ell}+1} y(x y)^{\bar{\ell}} \otimes y(x y)^{k-1} \cdot h_{1},
$$

we may assume that $\varphi_{\bar{\ell}+1}=0$. Consequently, using (2.35) and (2.36), we obtain additionally that $\beta_{k}=\beta$ and $c_{\bar{\ell}}=\widetilde{\beta}$; by symmetry, we have $\widetilde{\beta}_{k}=\widetilde{\beta}$ and $\widetilde{c}_{\bar{\ell}}=\beta$. Similar 
arguments, by symmetry, apply to the homogeneous component $\widetilde{q}^{(t)}$. Thus,

$$
\left(\begin{array}{c}
q^{(t)} \\
\widetilde{q}^{(t)}
\end{array}\right)=\left(\begin{array}{l}
\beta \cdot \sum_{i=\bar{\ell}+1}^{k}(x y)^{i} \otimes y(x y)^{\ell-i}+\widetilde{\beta} \cdot \sum_{i=\bar{\ell}}^{k-1} y(x y)^{i} \otimes(y x)^{\ell-i} \\
\widetilde{\beta} \cdot \sum_{i=\bar{\ell}+1}^{k}(y x)^{i} \otimes x(y x)^{\ell-i}+\beta \cdot \sum_{i=\bar{\ell}}^{k-1} x(y x)^{i} \otimes(x y)^{\ell-i}
\end{array}\right) .
$$

Replacing $s$ with the element

$$
s+\beta\left(x \otimes(x y)^{\bar{\ell}+1}-y(x y)^{k-1} \otimes y(x y)^{\bar{\ell}}\right) \cdot h_{1},
$$

we may assume that for the initial $s$ we have $\beta=0$. By symmetry, we may also assume that $\widetilde{\beta}=0$.

Step 6. Consider the case where $t=4 k-1$. We represent the homogeneous components $q^{(t)}$ and $\widetilde{q}^{(t)}$ in the form

$$
\begin{aligned}
q^{(t)}= & \alpha(x y)^{k} \otimes x(y x)^{k-1}+\beta(x y)^{k} \otimes y(x y)^{k-1} \\
& +a x(y x)^{k-1} \otimes(x y)^{k}+c y(x y)^{k-1} \otimes(x y)^{k}, \\
\widetilde{q}^{(t)}= & \widetilde{\alpha}(y x)^{k} \otimes y(x y)^{k-1}+\widetilde{\beta}(y x)^{k} \otimes x(y x)^{k-1} \\
& +\widetilde{a} y(x y)^{k-1} \otimes(y x)^{k}+\widetilde{c} x(y x)^{k-1} \otimes(y x)^{k}
\end{aligned}
$$

with $\alpha, \beta, a, c, \widetilde{\alpha}, \widetilde{\beta}, \widetilde{a}, \widetilde{c} \in K$. Equation (2.5) implies a unique relation

$$
\beta-c+\widetilde{\beta}-\widetilde{c}=0 .
$$

As at Step 1, using an analog of (2.18) we may assume that $a=0$. Moreover, as at Step 5 , replacing $s$ with the element of the form (2.37), where $\bar{\ell}=k-1$, we may assume that $\alpha=0$. By symmetry, $\widetilde{a}=0=\widetilde{\alpha}$. Next, replacing $s$ by $s-c \cdot y(x y)^{k-1} \otimes y(x)^{k-1} \cdot h_{1}$, we may assume additionally that $c=0$ and, by symmetry, also $\widetilde{c}=0$. Then $\beta+\widetilde{\beta}=0$; replacing $s$ with $s-\beta(x y)^{k} \otimes x \cdot h_{1}$, we may also assume that $\beta=0=\widetilde{\beta}$.

Step 7. Finally, if $t=4 k$, then

$\left(\begin{array}{c}q^{(t)} \\ \widetilde{q}^{(t)}\end{array}\right)=\left(\begin{array}{c}\alpha(x y)^{k} \otimes(x y)^{k} \\ \widetilde{\alpha}(x y)^{k} \otimes(x y)^{k}\end{array}\right)=\alpha \cdot(x y)^{k} \otimes y(x y)^{k-1} \cdot h_{1}+\widetilde{\alpha} \cdot(x y)^{k} \otimes x(y x)^{k-1} \cdot h_{2} \in \operatorname{Im} d_{1}$,

where $\alpha, \widetilde{\alpha} \in K$. A suitable replacement allows us to assume that for the initial $s$ we have $q^{(t)}=\widetilde{q}^{(t)}=0$.

We return to the proof of Theorem 2.1. Consider the following sequence in the category of right $\Lambda$-modules:

$$
\Lambda \stackrel{\widetilde{d}_{0}}{\longleftarrow} \Lambda^{2} \stackrel{\widetilde{d}_{1}}{\longleftarrow} \Lambda^{2}
$$

with

$$
\widetilde{d}_{0}=(*(x \otimes 1-1 \otimes x), \quad *(y \otimes 1-1 \otimes y)),
$$

where the matrix of the homomorphism $\widetilde{d}_{1}$ is the transpose of the matrix of $d_{1}$ (see (2.3)); the entries of the matrix of $\widetilde{d}_{1}$ determine the corresponding homomorphisms of multiplication by these elements from the left (cf. the notation in (2.39)). Using the right-left-symmetric version of Lemma 2.4 , we see that $\operatorname{Ker} \widetilde{d}_{0}=\operatorname{Im} \widetilde{d}_{1}$. To the sequence (2.38), we apply the duality functor

$$
D=\operatorname{Hom}_{K}(-, K): \operatorname{Mod}-\Lambda \longrightarrow \Lambda-\operatorname{Mod} .
$$

Clearly, $D\left(\widetilde{d}_{1}\right)=d_{1}$ and $D\left(\widetilde{d}_{0}\right)=d_{2}$, whence, by the exactness of the functor, we obtain $\operatorname{Ker} d_{1}=\operatorname{Im} d_{2}$.

Proposition 2.5. $\operatorname{Im} d_{3}=\Lambda g \simeq R$. 
Proof. First, we prove that $\operatorname{dim}_{K}(\Lambda g)=4 k$. Since $(x \otimes 1) g=(1 \otimes x) g$ and $(y \otimes 1) g=$ $(1 \otimes y) g$, the vector space $\Lambda g$ is spanned by the elements

$$
\begin{aligned}
\left((x y)^{i} \otimes 1\right) g,\left((y x)^{i} \otimes 1\right) g & (1 \leq i \leq k-1), \\
\left(x(y x)^{i} \otimes 1\right) g,\left(y(x y)^{i} \otimes 1\right) g & (0 \leq i \leq k-1),
\end{aligned}
$$

together with $g$ and $\left((x y)^{k} \otimes 1\right) g$. Clearly, all these elements are nonzero. Suppose that this set is linearly dependent. This means that there is a nonzero element $r \in R$ such that $(r \otimes 1) g=0$. Using the grading on $\Lambda$ introduced above and the fact that $g$ is a homogeneous element (of degree $2 k$ ), we may assume that $r$ is homogeneous. If $\operatorname{deg} r \in\{0,2 k\}$, we obtain a contradiction immediately. If $\operatorname{deg} r=2 i$ with $1 \leq i \leq k-1$, then the corresponding two elements in (2.40) are linearly dependent. However, the decompositions of these elements in the standard basis of $\Lambda$, namely,

$$
\begin{aligned}
& \left((x y)^{i} \otimes 1\right) g=\sum_{t=i}^{k}(x y)^{t} \otimes(x y)^{k+i-t}+\sum_{t=i}^{k-1}(x y)^{t} x \otimes y(x y)^{k+i-t-1}, \\
& \left((y x)^{i} \otimes 1\right) g=\sum_{t=i}^{k}(y x)^{t} \otimes(x y)^{k+i-t}+\sum_{t=i}^{k-1}(y x)^{t} y \otimes x(y x)^{k+i-t-1},
\end{aligned}
$$

show that they are linearly independent. Similarly, using the decompositions of elements in (2.41) (with fixed $i$ ), we obtain a contradiction again.

Now we consider a $\Lambda$-homomorphism $\psi: \Lambda \rightarrow \Lambda g$ such that $\psi(1 \otimes 1)=g$. Since

$$
\operatorname{Ker} \psi \supset_{\Lambda}\langle x \otimes 1-1 \otimes x, y \otimes 1-1 \otimes y\rangle=\operatorname{Ker} \mu,
$$

there exists an epimorphism $\psi^{\prime}: R \rightarrow \Lambda g$ with $\psi^{\prime} \circ \mu=\psi$. Since $\operatorname{dim}_{K} \Lambda g=4 k, \psi^{\prime}$ is an isomorphism.

To complete the proof of Theorem 2.1, it remains to show that the complex (2.2) is exact at the member $Q_{3}$, i.e., that $\operatorname{dim}_{K} \operatorname{Ker} d_{2}=\operatorname{dim}_{K} \operatorname{Im} d_{3}$. We have already proved the exactness of the sequence

$$
0 \longleftarrow R \stackrel{\mu}{\longleftarrow} Q_{0} \stackrel{d_{0}}{\longleftarrow} Q_{1} \stackrel{d_{1}}{\longleftarrow} Q_{2} \stackrel{d_{2}}{\longleftarrow} Q_{3} \longleftarrow \operatorname{Ker} d_{2} \longleftarrow 0 ;
$$

it follows that

$$
\operatorname{dim}_{K} R+\operatorname{dim}_{K} Q_{1}+\operatorname{dim}_{K} Q_{3}=\operatorname{dim}_{K} Q_{0}+\operatorname{dim}_{K} Q_{2}+\operatorname{dim}_{K} \operatorname{Ker} d_{2} .
$$

Finally, we obtain

$$
\operatorname{dim}_{K} \operatorname{Ker} d_{2}=\operatorname{dim}_{K} R=\operatorname{dim}_{K} \operatorname{Im} d_{3} .
$$

\section{§3. Additive structure of the cohomology algebra}

Let $Q . \stackrel{\mu}{\longrightarrow} R$ be the minimal $\Lambda$-projective resolution described in $₫ 2$, Then we have

$$
\operatorname{HH}^{n}(R)=\operatorname{Ext}_{\Lambda}^{n}(R, R)=\mathrm{H}^{n}\left(\operatorname{Hom}_{\Lambda}\left(Q_{\bullet}, R\right)\right),
$$

where $\operatorname{Hom}_{\Lambda}(Q \bullet, R)$ is the complex

$$
\left(\operatorname{Hom}_{\Lambda}\left(Q_{n}, R\right), \delta^{n}=\operatorname{Hom}_{\Lambda}\left(d_{n}, R\right)\right)_{n \geq 0} .
$$

Let $Z^{n}(R)$ (respectively, $B^{n}(R)$ ) denote the vector space of $n$-cocycles (respectively, $n$-coboundaries), i.e., $Z^{n}(R)=\operatorname{Ker} \delta^{n}, B^{n}(R)=\operatorname{Im} \delta^{n-1}$.

Since for any $n \geq 0$ the module $Q_{n}$ is isomorphic either to $\Lambda$ or to $\Lambda^{2}$, any element in $\operatorname{Hom}_{\Lambda}\left(Q_{n}, R\right)$ can be identified either with an element of $R$, or with a pair of elements of 
$R$, respectively. Upon this identification, the differential $\delta^{0}: R \rightarrow R^{2}$ can be described as follows: for any $r \in R$,

$$
\delta^{0}(r)=((x \otimes 1-1 \otimes x) r,(y \otimes 1-1 \otimes y) r)=(x r-r x, y r-r y) .
$$

Proposition 3.0.1. $\operatorname{dim}_{K} \mathrm{HH}^{0}(R)=k+3, \operatorname{dim} B^{1}(R)=3(k-1)$.

Proof. By [12, III.14], the center $Z(R)=Z^{0}(R)$ of the algebra $R$ admits the following basis:

$$
\left\{1, x y+y x,(x y)^{2}+(y x)^{2}, \ldots,(x y)^{k-1}+(y x)^{k-1}, x(y x)^{k-1}, y(x y)^{k-1},(x y)^{k}\right\} .
$$

Therefore, $\operatorname{dim}_{K} \mathrm{HH}^{0}(R)=k+3$ and $\operatorname{dim} B^{1}(R)=\operatorname{dim}_{K} R-\operatorname{dim}_{K} Z^{0}(R)=3(k-1)$.

Remark 3.0.2. In the sequel, for some of the elements in (3.3) we use an abbreviated notation:

$$
p_{1}:=x y+y x, \quad p_{2}:=x(y x)^{k-1}, \quad p_{2}^{\prime}:=y(x y)^{k-1}, \quad p_{3}:=(x y)^{k} .
$$

Observe that $(x y)^{i}+(y x)^{i}=p_{1}^{i}$ with $1 \leq i \leq k-1$. Moreover, if char $K \neq 2$, then $(x y)^{k}=\frac{1}{2} p_{1}^{k}$.

Remark 3.0.3. Letting $r$ in (3.2) run over the standard basis of the algebra $R$, we easily see that $B^{1}(R)$ admits the following basis:

$$
\left((x y)^{i}-(y x)^{i}, 0\right),\left(0,(y x)^{i}-(x y)^{i}\right),\left(x(y x)^{i},-y(x y)^{i}\right), \quad \text { where } 1 \leq i \leq k-1 .
$$

3.1. The differential $\delta^{1}$. To study the differential $\delta^{1}: R^{2} \rightarrow R^{2}$, we need to impose additional assumptions on the parameter $k$ (see (1.1)) and on $p=\operatorname{char} K$.

Case 1: $k \geq 3$. In this case the condition $\left(r_{1}, r_{2}\right) \in Z^{1}(R)$ is equivalent to the following system of equations (over $R$ ):

$$
\left\{\begin{array}{r}
x r_{1}+r_{1} x-\sum_{i=0}^{k-2}(y x)^{i} y \cdot r_{1} \cdot y(x y)^{k-2-i}-\sum_{i=0}^{k-1}(y x)^{i} \cdot r_{2} \cdot(x y)^{k-1-i}=0 \\
-\sum_{i=0}^{k-1}(x y)^{i} \cdot r_{1} \cdot(y x)^{k-1-i}+y r_{2}+r_{2} y-\sum_{i=0}^{k-2}(x y)^{i} x \cdot r_{2} \cdot x(y x)^{k-2-i}=0 .
\end{array}\right.
$$

Consider the decompositions of $r_{1}$ and $r_{2}$ in the standard basis of the algebra $R$ :

$$
r_{1}=\sum_{b \in \mathcal{B}} \lambda_{b} b, \quad r_{2}=\sum_{b \in \mathcal{B}} \mu_{b} b
$$

here $\mathcal{B}:=\mathcal{B}_{\text {st }}$ and $\lambda_{b}, \mu_{b} \in K$. Substituting this in the first equation in (3.5), we obtain the relations

$$
\begin{aligned}
2 \lambda_{1}=0, & \mu_{1}=\lambda_{y(x y)^{k-2}} ; \\
\lambda_{y(x y)^{i-1}}=0 & \text { for } 1 \leq i \leq k-2 ; \\
\lambda_{(y x)^{i}}+\lambda_{(x y)^{i}}=0 & \text { for } 1 \leq i \leq k-1 ; \\
(3-k) \lambda_{x}= & k \mu_{y} ; \\
2 \lambda_{y(x y)^{k-1}} & =\mu_{x y}+\mu_{y x} .
\end{aligned}
$$


By symmetry, the second equation in (3.5) implies

$$
\begin{aligned}
2 \mu_{1}=0, & \lambda_{1}=\mu_{x(y x)^{k-2}} ; \\
\mu_{x(y x)^{i-1}}=0 & \text { for } 1 \leq i \leq k-2 ; \\
\mu_{(x y)^{i}}+\mu_{(y x)^{i}}=0 & \text { for } 1 \leq i \leq k-1 ; \\
(3-k) \mu_{y}= & k \lambda_{x} ; \\
2 \mu_{x(y x)^{k-1}} & =\lambda_{y x}+\lambda_{x y} .
\end{aligned}
$$

It should be noted that the scalars $\lambda_{x(y x)^{i}}$ and $\mu_{y(x y)^{i}}$ with $1 \leq i \leq k-1$ do not occur in these two groups of relations, so that the elements in $\operatorname{Hom}_{\Lambda}\left(Q_{1}, R\right)=R^{2}$ of the form

$$
\left(x(y x)^{i}, 0\right),\left(0, y(x y)^{i}\right) \quad \text { for } 1 \leq i \leq k-1
$$

lie in $Z^{1}(R)$.

Next, (3.9) and (3.11) yield

$$
2 \lambda_{y(x y)^{k-1}}=0 .
$$

By symmetry,

$$
2 \mu_{x(y x)^{k-1}}=0 .
$$

Moreover, from (3.8) and (3.12) it follows that the scalars $\lambda_{x}$ and $\mu_{y}$ satisfy a homogeneous system of linear equations (over $K$ ) with the matrix

$$
C=\left(\begin{array}{cc}
3-k & -k \\
-k & 3-k
\end{array}\right) .
$$

Case 1.1: $p=2$. We continue to study system (3.5) under the additional assumption char $K=2$.

Proposition 3.1.1. If $p=2$ and $k \geq 3$, then a $K$-basis of the vector space $\operatorname{HH}^{1}(R)$ is formed by the cohomology classes of the following elements in $\operatorname{Hom}_{\Lambda}\left(Q_{1}, R\right)=R^{2}$ :

$$
\begin{aligned}
& \left(1, x(y x)^{k-2}\right), \quad\left(y(x y)^{k-2}, 1\right) ; \\
& \left(x(y x)^{i}, 0\right) \quad \text { for } 1 \leq i \leq k-1 ; \\
& \left(y(x y)^{k-1}, 0\right), \quad\left(0, x(y x)^{k-1}\right) ; \\
& \left((x y)^{k}, 0\right), \quad\left(0,(x y)^{k}\right) .
\end{aligned}
$$

Proof. Now we have $\operatorname{det} C=1$, whence $\lambda_{x}=0=\mu_{y}$. Arguing as above, we easily show that as a $K$-basis of the vector space $Z^{1}(R)$ we can take the set formed by the elements listed in (3.13), (3.16), (3.18), and (3.19), and also by the elements

$$
\left((x y)^{i}-(y x)^{i}, 0\right),\left(0,(y x)^{i}-(x y)^{i}\right) \quad \text { for } 1 \leq i \leq k-1 .
$$

Combining this and Remark 3.0.3, we arrive at the desired statement.

Corollary 3.1.2. If $p=2$ and $k \geq 3$, then

$$
\operatorname{dim}_{K} Z^{1}(R)=4 k+2, \quad \operatorname{dim}_{K} H H^{1}(R)=k+5, \quad \operatorname{dim}_{K} B^{2}(R)=4 k-2 .
$$


Remark 3.1.3. It is verified directly that the vector space $B^{2}(R)$ is generated by the following elements:

$$
\begin{gathered}
\left((x y)^{i}+(y x)^{i}, 0\right),\left(0,(y x)^{i}+(x y)^{i}\right) \quad \text { for } 1 \leq i \leq k-1, \\
\left(x(y x)^{i}, 0\right),\left(0, y(x y)^{i}\right) \quad \text { for } 2 \leq i \leq k-1, \\
\delta^{1}(x, 0)=\left((3-k) y(x y)^{k-1},-K B(y x)^{k-1}\right), \\
\delta^{1}(0, y)=\left(-k y(x y)^{k-1},(3-k) x(y x)^{k-1}\right), \\
\delta^{1}(x y, 0)=\left(x y x,-(x y)^{k}\right), \quad \delta^{1}(0, y x)=\left(-(x y)^{k}, y x y\right) .
\end{gathered}
$$

Since the number of these elements is equal to $4 k-2$, they form a basis of $B^{2}(R)$. Furthermore, $\operatorname{det} C \neq 0$, so that we can replace $\delta^{1}(x, 0)$ and $\delta^{1}(0, y)$ in this basis by the elements

$$
\left(y(x y)^{k-1}, 0\right) \text { and }\left(0, x(y x)^{k-1}\right) .
$$

Remark 3.1.4. In the sequel, if $f \in Z^{n}(R)$ is an $n$-cocycle, its cohomology class is often denoted still by $f$.

Case 1.2: $p=3$. Relations (3.7), (3.10), (3.14), and (3.15) imply that

$$
\lambda_{1}=0=\mu_{1}, \quad \lambda_{y(x y)^{k-2}}=0=\mu_{x(y x)^{k-2}}, \quad \lambda_{y(x y)^{k-1}}=0=\mu_{x(y x)^{k-1}} .
$$

Now we consider two subcases.

Case 1.2a: Assume additionally that 3 does not divide $k$.

Proposition 3.1.5. If $p=3, k \geq 3$, and 3 does not divide $k$, then a $K$-basis of the vector space $\mathrm{HH}^{1}(R)$ is formed by the cohomology classes of the elements listed in (3.17) and (3.19), together with the cohomology class of the element $(x,-y)$.

Proof. In this case we have $\operatorname{rk} C=1$; hence, the homogeneous system of linear equations with the matrix $C$ reduces to a single equation, namely, to $\lambda_{x}+\mu_{y}=0$, whence we see that $(x,-y) \in Z^{1}(R)$. The above arguments show that the elements occurring in (3.13), (3.19), and (3.20), together with $(x,-y)$, form a basis of $Z^{1}(R)$. Now, Remark 3.0.3 completes the proof of Proposition 3.1.5.

Corollary 3.1.6. If $p=3, k \geq 3$, and 3 does not divide $k$, then

$$
\operatorname{dim}_{K} Z^{1}(R)=4 k-1, \quad \operatorname{dim}_{K} H H^{1}(R)=k+2, \quad \operatorname{dim}_{K} B^{2}(R)=4 k+1 .
$$

Remark 3.1.7. It is easily seen that, in the case under consideration (i.e., $p=3$ does not divide $k$ and $k \geq 3$ ), the vector space $B^{2}(R)$ is generated by the set consisting of the elements occurring in (3.21) and the elements

$$
\begin{gathered}
\delta^{1}(1,0)=\left(2 x,-(y x)^{k-1}-(x y)^{k-1}\right), \quad \delta^{1}(0,1)=\left(-(x y)^{k-1}-(y x)^{k-1}, 2 y\right), \\
-\frac{1}{k} \delta^{1}(x, 0)=\left(y(x y)^{k-1}, x(y x)^{k-1}\right), \\
\frac{1}{2} \delta^{1}\left(y(x y)^{k-1}, 0\right)=\left((x y)^{k}, 0\right), \quad \frac{1}{2} \delta^{1}\left(0, x(y x)^{k-1}\right)=\left(0,(x y)^{k}\right) .
\end{gathered}
$$

This set is a basis of $B^{2}(R)$, because its cardinality is equal to $4 k+1$. Moreover, since $\left(0,(y x)^{k-1}+(x y)^{k-1}\right),\left((x y)^{k-1}+(y x)^{k-1}, 0\right) \in B^{2}(R)$, the elements $\delta^{1}(1,0)$ and $\delta^{1}(0,1)$ can be replaced in this basis with $(x, 0)$ and $(0, y)$.

Case 1.2b: 3 divides $k$.

Proposition 3.1.8. If $p=3$ and 3 divides $k$, then a $K$-basis of the vector space $\operatorname{HH}^{1}(R)$ is formed by the cohomology classes of the elements occurring in (3.17) and (3.19), together with the cohomology classes of the elements $(x, 0)$ and $(0, y)$. 
Proof. Now we have $\operatorname{rk} C=0$; hence, to get a basis of the vector space $Z^{1}(R)$, we need to adjoin the elements $(x, 0)$ and $(0, y)$ to the elements listed in (3.13), (3.19), and (3.20). Then the proof is completed in the same way as that of Proposition 3.1.5.

Corollary 3.1.9. If $p=3$ and 3 divides $k$, then

$$
\operatorname{dim}_{K} Z^{1}(R)=4 k, \quad \operatorname{dim}_{K} H H^{1}(R)=k+3, \quad \operatorname{dim}_{K} B^{2}(R)=4 k .
$$

Remark 3.1.10. Under the assumptions of Proposition 3.1.8 a basis of $B^{2}(R)$ is obtained by deleting the element $\left(y(x y)^{k-1}, x(y x)^{k-1}\right)$ from the set indicated in Remark 3.1.7 (because now we have $\delta^{1}(x, 0)=0$; see (3.22) ).

Case 1.3: $p \notin\{2,3\}$. Again we consider two subcases.

Case 1.3a: Assume additionally that $3-2 k \neq 0$ (in the field $K$ ), i.e., $\operatorname{rk} C=2$.

Proposition 3.1.11. If $p \notin\{2,3\}, k \geq 3$, and $p$ does not divide $3-2 k$, then a $K$-basis of the vector space $\mathrm{HH}^{1}(R)$ is formed by the cohomology classes of the elements occurring in (3.17) and (3.19).

Proof. First, relations (3.24) are satisfied since $p \neq 2$ (cf. case 1.2). Moreover, we have $\operatorname{rk} C=2$, whence $\lambda_{x}=0=\mu_{y}$. Consequently, the vector space $Z^{1}(R)$ admits a basis consisting of the elements of the form (3.13), (3.19), and (3.20). Using Remark 3.0.3, we obtain the desired statement.

Corollary 3.1.12. If $p \notin\{2,3\}, k \geq 3$, and $p$ does not divide $3-2 k$, then

$$
\operatorname{dim}_{K} Z^{1}(R)=4 k-2, \quad \operatorname{dim}_{K} H H^{1}(R)=k+1, \quad \operatorname{dim}_{K} B^{2}(R)=4 k+2 .
$$

Remark 3.1.13. Under the assumptions of Proposition 3.1.11, the vector space $B^{2}(R)$ is generated by the set of elements indicated in (3.21) and (3.26), together with the elements $\delta^{1}(x, 0), \delta^{1}(0, y)$ (see (3.22) and (3.23) $)$. Since the cardinality of this set is equal to $4 k+2$, this is a basis of $B^{2}(R)$. Moreover, since $\mathrm{rk} C=2$, we see that the elements $\delta^{1}(x, 0)$ and $\delta^{1}(0, y)$ can be replaced in this basis with the elements $\left(y(x y)^{k-1}, 0\right)$ and $\left(0, x(y x)^{k-1}\right)$.

Case 1.3b: Assume that $3-2 k=0$ in $K$. Clearly, in this case $p$ does not divide $k$ and $\operatorname{rk} C=1$.

Proposition 3.1.14. If $p \notin\{2,3\}$ and $p$ divides $3-2 k$, then a $K$-basis of the vector space $\mathrm{HH}^{1}(R)$ is formed by the cohomology classes of the elements occurring in (3.17) and (3.19), together with the cohomology class of the element $(x, y)$.

Proof. Since rk $C=1$, the homogeneous system of linear equations with the matrix $C$ reduces to a single equation, namely, to $\lambda_{x}-\mu_{y}=0$, whence we see that $(x, y) \in Z^{2}(R)$. Now the proof can be completed like that of Proposition 3.1.5.

Corollary 3.1.15. If $p \notin\{2,3\}$ and $p$ divides $3-2 k$, then

$$
\operatorname{dim}_{K} Z^{1}(R)=4 k-1, \quad \operatorname{dim}_{K} H H^{1}(R)=k+2, \quad \operatorname{dim}_{K} B^{2}(R)=4 k+1 .
$$

Remark 3.1.16. Under the assumptions of Proposition 3.1.14 the vector space $B^{2}(R)$ is generated by the set indicated in Remark 3.1.7. To see this, we need a minor modification in the corresponding argument; in fact, now we have (cf. (3.25))

$$
-\frac{1}{k} \delta^{1}(x, 0)=\left(-y(x y)^{k-1}, x(y x)^{k-1}\right) .
$$


Case 2: $k=2$. If $k=2$, then the condition $\delta^{1}\left(r_{1}, r_{2}\right)=0$ implies the following relations for coefficients in the decompositions of the elements $r_{1}$ and $r_{2}$ (see (3.6)):

$$
\begin{gathered}
2 \lambda_{1}=0=\mu_{1}, \quad \lambda_{y}=\mu_{1}, \quad \mu_{x}=\lambda_{1}, \\
\lambda_{1}=\lambda_{x y}+\lambda_{y x}, \quad \mu_{1}=\mu_{x y}+\mu_{y x} ; \\
\left\{\begin{array}{l}
\lambda_{x}-2 \mu_{y}=0, \\
2 \lambda_{x}-\mu_{y}=0 ;
\end{array}\right. \\
\left\{\begin{array}{l}
\lambda_{y}+\mu_{x y}+\mu_{y x}=2 \lambda_{y x y}, \\
\mu_{x}+\lambda_{x y}+\lambda_{y x}=2 \mu_{x y x} .
\end{array}\right.
\end{gathered}
$$

Case 2.1: $p=2$. We continue the analysis of the above relations under the additional assumption that $p=2$.

Proposition 3.1.17. If $p=2$ and $k=2$, then a $K$-basis of the vector space $\operatorname{HH}^{1}(R)$ is formed by the cohomology classes of the elements

$$
(1+x y, x),(y, 1+y x),(y x y, 0),(0, x y x),(x y x, 0),\left((x y)^{2}, 0\right),\left(0,(x y)^{2}\right) .
$$

Proof. If $p=2$, then (3.27) implies $\lambda_{x}=0=\mu_{y}$, while (3.28) is a consequence of the remaining relations and can be omitted. It follows that, as a basis for $Z^{1}(R)$, we can take the set of elements listed in (3.29) together with

$$
(x y-y x, 0),(0, y x-x y),(0, y x y) .
$$

Using Remark 3.0.3, we arrive at the desired statement.

Remark 3.1.18. The proof of Proposition 3.1.17 shows that the formulas for the dimensions of the vector spaces $Z^{1}(R), H H^{1}(R)$, and $B^{2}(R)$, as indicated in Corollary 3.1.2, remain valid in the case in question. Moreover, we can argue as in Remark 3.1.3 to prove that, as a basis for $B^{2}(R)$, we can take the set of the following elements:

$$
(x y+y x, y x y),(x y x, y x+x y),(y x y, 0),(0, x y x),\left(x y x,(x y)^{2}\right),\left((x y)^{2}, y x y\right) .
$$

Case 2.2: $p \neq 2$. If $p \neq 2$ (and $k=2$ ), then it is easily seen that the arguments of Case 1.2 (for $p=3$ ) and Case 1.3 (for $p \notin\{2,3\}$ ) apply without change.

3.2. The differential $\delta^{2}$. In this subsection we study the differential $\delta^{2}: R^{2} \rightarrow R$, where

$$
\delta^{2}\left(r_{1}, r_{2}\right)=x r_{1}-r_{1} x+y r_{2}-r_{2} y,
$$

and also we describe $\mathrm{HH}^{2}(R)$.

Proposition 3.2.1. a) The vector space $Z^{2}(R)$ admits a $K$-basis formed by the following elements:

$$
\begin{gathered}
\left(y(x y)^{i}, x(y x)^{i}\right) \quad \text { for } 0 \leq i \leq k-2 \\
\left((x y)^{i}+(y x)^{i}, 0\right),\left(0,(y x)^{i}+(x y)^{i}\right),\left(x(y x)^{i}, 0\right),\left(0, y(x y)^{i}\right) \quad \text { for } 1 \leq i \leq k-1 \\
(1,0),(0,1),(x, 0),(0, y) \\
\left(y(x y)^{k-1}, 0\right),\left(0, x(y x)^{k-1}\right) \\
\left((x y)^{k}, 0\right),\left(0,(x y)^{k}\right)
\end{gathered}
$$

b) The vector space $B^{3}(R)$ admits a K-basis formed by the elements

$$
(x y)^{i}-(y x)^{i}, x(y x)^{i}, y(x y)^{i} \quad \text { for } 1 \leq i \leq k-1 .
$$


Proof. Looking at decompositions of the form (3.6) in the standard basis of $R$, we see that the relation $\delta^{2}\left(r_{1}, r_{2}\right)=0$ is equivalent to the following equations for the coefficients $\lambda_{b}, \mu_{b}\left(b \in \mathcal{B}_{s t}\right)$ of these decompositions:

$$
\begin{gathered}
\lambda_{y(x y)^{i}}=\mu_{x(y x)^{i}} \quad \text { for } 0 \leq i \leq k-2 \\
\lambda_{(y x)^{i}}=\lambda_{(x y)^{i}}, \mu_{(x y)^{i}}=\mu_{(y x)^{i}} \quad \text { for } 1 \leq i \leq k-1 .
\end{gathered}
$$

This implies directly that the set of elements listed in (3.30)-(3.34) is a basis of $Z^{2}(R)$. In particular, we have $\operatorname{dim}_{K} Z^{2}(R)=5 k+3, \operatorname{dim}_{K} B^{3}(R)=3 k-3$.

Next, computing all elements of the form $\delta^{2}(b, 0)$ and $\delta^{2}(0, b)$ with $b \in \mathcal{B}_{\text {st }}$, we see that the elements in (3.35) generate $B^{3}(R)$. Since their number is equal to $\operatorname{dim}_{K} B^{3}(R)$, they form a basis of this vector space.

Corollary 3.2.2. a) $\operatorname{dim}_{K} Z^{2}(R)=5 k+3, \operatorname{dim}_{K} B^{3}(R)=3 k-3$.

b)

$$
\operatorname{dim}_{K} \mathrm{HH}^{2}(R)= \begin{cases}k+5 & \text { if } p=2, \\ k+3 & \text { if } p=3 \text { and } 3 \text { divides } k, \\ k+2 & \text { if } p=3 \text { and } 3 \text { does not divide } k, \\ & \text { or } p \notin\{2,3\} \text { and } p \text { divides } 3-2 k, \\ k+1 & \text { if } p \notin\{2,3\} \text { and } p \text { does not divide } 3-2 k .\end{cases}
$$

Proof. Statement a) follows from Proposition 3.2.1. Statement b) follows from Corollaries 3.1.2 3.1.9, 3.1.12, and 3.1.15, and from Remark 3.1.18,

Proposition 3.2.3. a) If $p=2$, then a $K$-basis of the vector space $\mathrm{HH}^{2}(R)$ is formed by the cohomology classes of the elements occurring in (3.30), (3.32), and (3.34).

b) Let $p=3$. If 3 does not divide $k$, then a $K$-basis of the vector space $\mathrm{HH}^{2}(R)$ is formed by the cohomology classes of the elements (3.30) and the cohomology classes of the elements

$$
(1,0),(0,1),\left(y(x y)^{k-1}, 0\right) .
$$

If 3 divides $k$, we should add the element $\left(0, x(y x)^{k-1}\right)$ to the preceding set in order to get a basis of $\mathrm{HH}^{2}(R)$.

c) Assume that $p \notin\{2,3\}$. If $p$ divides $3-2 k$, then a $K$-basis for the vector space $\mathrm{HH}^{2}(R)$ is formed by the set of the cohomology classes of the elements listed in (3.30) and (3.36). If $p$ does not divide $3-2 k$, then we should remove the element $\left(y(x y)^{k-1}, 0\right)$ from the preceding set in order to get a basis of $\mathrm{HH}^{2}(R)$.

Proof. In all cases, the statements follow (with the help of Corollary 3.2.2) immediately from Proposition 3.2.1 and Remarks 3.1.3, 3.1.7, 3.1.10, 3.1.13, 3.1.16, and 3.1.18, in which the corresponding bases for $B^{2}(R)$ were presented.

3.3. The differential $\delta^{3}$. Since the resolution (2.2) is 4-periodic, it remains to study the differential $\delta^{3}: R \rightarrow R$. Since $\delta^{3}={ }^{*} g$, we have

$$
\begin{aligned}
\delta^{3}(r)= & \sum_{i=1}^{k}(y x)^{i} \cdot r \cdot(y x)^{k-i}+\sum_{i=0}^{k-1} y(x y)^{i} \cdot r \cdot x(y x)^{k-1-i} \\
& +\sum_{i=0}^{k-1} x(y x)^{i} \cdot r \cdot y(x y)^{k-1-i}+\sum_{i=0}^{k-1}(x y)^{i} \cdot r \cdot(x y)^{k-i} .
\end{aligned}
$$

Proposition 3.3.1. a) If $p$ does not divide $2 k$, then the set $\mathcal{B}_{\text {st }} \backslash\{1\}$ is a basis for $Z^{3}(R)$.

b) If $p$ divides $2 k$, then $\delta^{3}=0$, whence $Z^{3}(R)=R$. 
Proof. Since $g$ is a homogeneous element of degree $2 k$ (see (2.4)), for any $b \in \mathcal{B}_{\text {st }} \backslash\{1\}$ we have $\delta^{3}(b)=0$. Moreover, relation (3.37) implies $\delta^{3}(1)=4 k(x y)^{k}$. Now, both statements follow immediately.

Proposition 3.3.2. a) If $p$ divides $2 k$, then a $K$-basis of the vector space $\mathrm{HH}^{3}(R)$ is formed by the cohomology classes of the following elements:

$$
\begin{cases} & 1, x, y \\ (x y)^{i} & \text { with } 1 \leq i \leq k .\end{cases}
$$

If $p$ does not divide $2 k$, then, in order to get a basis of $\mathrm{HH}^{3}(R)$, we should remove the element 1 from (3.38).

b) If $p$ divides $2 k$, then

$$
\mathrm{HH}^{4}(R) \simeq \mathrm{HH}^{0}(R)
$$

If $p$ does not divide $2 k$, then a $K$-basis for the vector space $\mathrm{HH}^{4}(R)$ is formed by the cohomology classes of the elements

$$
(x y)^{i}+(y x)^{i} \quad \text { for } 1 \leq i \leq k-1
$$

together with the cohomology classes of the elements $1, x(y x)^{k-1}, y(x y)^{k-1}$.

Proof. The first statement follows from Propositions 3.3.1,a) and 3.2.1,b); the second is a consequence of the proofs of Propositions 3.3.1 and 3.0.1

\section{Corollary 3.3.3.}

$$
\operatorname{dim}_{K} \mathrm{HH}^{3}(R)=\operatorname{dim}_{K} \mathrm{HH}^{4}(R)=\left\{\begin{array}{l}
k+3 \quad \text { if } p \text { divides } 2 k \\
k+2 \text { if } p \text { does not divide } 2 k .
\end{array}\right.
$$

\section{§4. Generators and Relations}

We briefly recall an interpretation of the Yoneda product in

$$
\mathrm{HH}^{*}(R)=\operatorname{Ext}_{\Lambda}^{*}(R, R)=\bigoplus_{m \geq 0} \operatorname{Ext}_{\Lambda}^{m}(R, R)
$$

which was used in [11].

Let $\mu: Q_{\bullet} \rightarrow R$ be the minimal $\Lambda$-projective resolution (see $\S 2$ ). Let $\operatorname{Hom}_{\Lambda}\left(Q_{\bullet}, R\right)=$ $\left(\operatorname{Hom}_{\Lambda}\left(Q_{n}, R\right), \delta^{n}\right)$ be the complex (3.1). Any $n$-cocycle $f \in Z^{n}(R)=\operatorname{Ker} \delta^{n}$ is lifted (uniquely up to homotopy) to a chain map of complexes $\left\{\varphi_{i}: Q_{n+i} \rightarrow Q_{i}\right\}_{i \geq 0}$. The homomorphism $\varphi_{i}$ is called the $i$ th translate of the cocycle $f$ and will be denoted by $\mathrm{T}^{i}(f)$. For cocycles $f \in \operatorname{Ker} \Delta^{n}$ and $g \in \operatorname{Ker} \Delta^{t}$, we have $\operatorname{cl} g \cdot \operatorname{cl} f=\operatorname{cl}\left(\mu \mathrm{T}^{0}(g) \mathrm{T}^{t}(f)\right)$. Consequently, for us it suffices to know the composition of the translates $\mathrm{T}^{0}(g)$ and $\mathrm{T}^{t}(f)$. In the sequel, we shall use direct decompositions of the modules $Q_{i}$ to describe the translates of cocycles and their products with the help of the matrices that correspond to such decompositions. Observe that, by the graded-commutativity of the algebra $\mathrm{HH}^{*}(R)$, to compute the product of a pair of homogeneous elements in $\mathrm{HH}^{*}(R)$ it suffices to know, for an element of degree $i$, its translates of order not exceeding $i$.

4.1. char $K=2$. In this subsection, we assume that the ground field $K$ has characteristic two, if not stipulated otherwise.

Case 1: $k \geq 3$. Consider the following homogeneous elements in $\operatorname{HH}^{*}(R)$ :

of degree $1: \quad u_{1}:=\left(1, x(y x)^{k-2}\right), u_{1}^{\prime}:=\left(y(x y)^{k-2}, 1\right), u_{2}:=(x y x, 0) ;$

of degree $2: \quad v_{1}:=(y, x), v_{2}:=(x, 0), v_{2}^{\prime}:=(0, y)$;

of degree $3: \quad w:=x y$;

of degree $4: \quad z:=1$. 
Recall that earlier we have distinguished (in the case of a field $K$ with arbitrary characteristic) certain elements in $\mathrm{HH}^{0}(R): p_{1}, p_{2}, p_{2}^{\prime}, p_{3}$ (see (3.4)).

Proposition 4.1.1. Assume that $p=2$ and $k \geq 3$. Then, in the algebra $\operatorname{HH}^{*}(R)$, the elements of the set

$$
\mathcal{Y}_{1}=\left\{p_{1}, p_{2}, p_{2}^{\prime}, p_{3}, u_{1}, u_{1}^{\prime}, u_{2}, v_{1}, v_{2}, v_{2}^{\prime}, w, z\right\}
$$

satisfy the following relations:

$$
\begin{aligned}
& \left\{\begin{array}{c}
p_{1}^{k}=p_{2}^{2}=\left(p_{2}^{\prime}\right)^{2}=p_{1} p_{2}=p_{1} p_{2}^{\prime}=p_{2} p_{2}^{\prime}=0 \\
p_{3}^{2}=p_{1} p_{3}=p_{2} p_{3}=p_{2}^{\prime} p_{3}=0
\end{array}\right. \\
& \left\{\begin{array}{c}
p_{1}^{k-1} u_{2}=p_{2} u_{2}=p_{2}^{\prime} u_{2}=p_{3} u_{2}=0 \\
p_{2} u_{1}=p_{2}^{\prime} u_{1}^{\prime}=p_{1}^{k-2} u_{2}
\end{array}\right. \\
& \left\{\begin{array}{c}
p_{2}^{\prime} u_{1}=p_{1} u_{1}^{\prime}, \quad p_{1} u_{1}=p_{2} u_{1}^{\prime}, \\
p_{1}^{k-1} v_{1}=p_{2} v_{2}=p_{2}^{\prime} v_{2}^{\prime}=p_{3} v_{1}=p_{3} v_{2}=p_{3} v_{2}^{\prime}=0 ;
\end{array}\right. \\
& p_{2} w=p_{2}^{\prime} w=p_{3} w=0 ; \\
& p_{2} v_{1}=p_{1} v_{2}^{\prime}=p_{2}^{\prime} v_{2}=p_{3} u_{1}^{2}, \quad p_{2}^{\prime} v_{1}=p_{1} v_{2}=p_{2} v_{2}^{\prime}=p_{3}\left(u_{1}^{\prime}\right)^{2} \text {; } \\
& u_{1} u_{1}^{\prime}=k p_{1}^{k-2} v_{1} \\
& u_{1} u_{2}=k p_{3}\left(u_{1}^{\prime}\right)^{2}, \quad u_{1}^{\prime} u_{2}=k p_{3} u_{1}^{2}, \quad u_{2}^{2}=0 ; \\
& u_{2} v_{2}=u_{2} v_{2}^{\prime}=0, \quad u_{2} v_{1}=p_{1} w, \quad u_{1}^{\prime} v_{2}=u_{1} v_{2}^{\prime}=p_{1}^{k-2} w ; \\
& u_{1} v_{1}=u_{1}^{\prime} v_{2}^{\prime}, \quad u_{1}^{\prime} v_{1}=u_{1} v_{2}, \quad u_{1}^{3}=\left(u_{1}^{\prime}\right)^{3} \text {; } \\
& v_{2}^{2}=\left(v_{2}^{\prime}\right)^{2}=v_{1} v_{2}=v_{1} v_{2}^{\prime}=v_{2} v_{2}^{\prime}=0 ; \\
& v_{1}^{2}=p_{1}^{2} z, \quad u_{1} w=u_{1}^{\prime} w=u_{2} w=v_{2} w=v_{2}^{\prime} w=0 ; \\
& v_{1} w=p_{1} u_{2} z, \quad w^{2}=0 .
\end{aligned}
$$

Proof. Relations (4.2) -(4.5) are verified directly. To prove the remaining relations, we need to compute the translates of suitable order for the elements in $\mathcal{Y}_{1}$ that have a positive degree. By the 4-periodicity of the minimal $\Lambda$-projective resolution (2.2), for the element $z$ the identity maps of the corresponding modules can be taken as the translates $\mathrm{T}^{i}(z)$.

Lemma 4.1.2. For the role of those translates of the elements in $\mathcal{Y}_{1} \backslash\{z\}$ that have a positive degree, we can take the homomorphisms determined by the following matrices:

$$
\begin{aligned}
\mathrm{T}^{0}\left(u_{1}\right) & =\left(1 \otimes 1, \quad x(y x)^{k-2} \otimes 1\right), \\
\mathrm{T}^{1}\left(u_{1}\right) & =\left(\begin{array}{cc}
1 \otimes 1+(x y)^{k-2} \otimes(x y)^{k-1}+(y x)^{k-2} \otimes(y x)^{k-1} & \sum_{i=0}^{k-2} y(x y)^{i} \otimes(y x)^{k-2-i} \\
\sum_{i=0}^{k-2}(y x)^{i} \otimes y(x y)^{k-2-i} & \star
\end{array}\right)
\end{aligned}
$$

with

$$
\mathrm{T}^{1}\left(u_{1}\right)_{2,2}=\sum_{i=1}^{k-2}(x y)^{i} \otimes x(y x)^{k-2-i}+x(y x)^{k-2} \otimes 1+\sum_{i=0}^{k-2}(y x)^{i} \otimes x(y x)^{k-2-i}
$$


at the position (2.2);

$$
\begin{aligned}
& \mathrm{T}^{0}\left(u_{1}^{\prime}\right)=\left(y(x y)^{k-2} \otimes 1, \quad 1 \otimes 1\right), \\
& \mathrm{T}^{1}\left(u_{1}^{\prime}\right)=\left(\begin{array}{cc}
\star & \sum_{i=0}^{k-2}(x y)^{i} \otimes x(y x)^{k-2-i} \\
\sum_{i=0}^{k-2} x(y x)^{i} \otimes(x y)^{k-2-i} & 1 \otimes 1+(y x)^{k-2} \otimes(y x)^{k-1}+(x y)^{k-2} \otimes(x y)^{k-1}
\end{array}\right)
\end{aligned}
$$

with

$$
\begin{aligned}
& \mathrm{T}^{1}\left(u_{1}^{\prime}\right)_{1,1}=\sum_{i=1}^{k-2}(y x)^{i} \otimes y(x y)^{k-2-i}+y(x y)^{k-2} \otimes 1+\sum_{i=0}^{k-2}(x y)^{i} \otimes y(x y)^{k-2-i} \\
& \mathrm{~T}^{0}\left(u_{2}\right)=(x y x \otimes 1, \quad 0), \\
& \mathrm{T}^{1}\left(u_{2}\right)=\left(\begin{array}{cc}
-x y x \otimes 1+\sum_{i=2}^{k-1}(i-1) y(x y)^{i} \otimes y(x y)^{k-1-i} & \sum_{i=2}^{k}(i-1)(x y)^{i} \otimes(y x)^{k-i} \\
\sum_{i=2}^{k}(i-1)(y x)^{i} \otimes(x y)^{k-i} & \sum_{i=1}^{k-1} i x(y x)^{i} \otimes x(y x)^{k-1-i}
\end{array}\right) ; \\
& \mathrm{T}^{0}\left(v_{1}\right)=(1 \otimes y, \quad x \otimes 1), \quad \mathrm{T}^{1}\left(v_{1}\right)=\left(\begin{array}{c}
y \otimes 1 \\
1 \otimes x
\end{array}\right), \\
& \mathrm{T}^{2}\left(v_{1}\right)=\left(\begin{array}{l}
-x \otimes x y+y(x y)^{k-1} \otimes y+x(y x)^{k-1} \otimes(y x)^{k-1} \\
-y \otimes y x+x(y x)^{k-1} \otimes x+y(x y)^{k-1} \otimes(x y)^{k-1}
\end{array}\right) ; \\
& \mathrm{T}^{0}\left(v_{2}\right)=(x \otimes 1, \quad 0), \quad \mathrm{T}^{1}\left(v_{2}\right)=\left(\begin{array}{c}
x \otimes 1 \\
0
\end{array}\right) \\
& \mathrm{T}^{2}\left(v_{2}\right)=\left(\begin{array}{c}
\sum_{i=1}^{k}(y x)^{i} \otimes(y x)^{k-i}+\sum_{i=0}^{k-1}(x y)^{i} \otimes(x y)^{k-i} \\
y \otimes y(x y)^{k-1}+x(y x)^{k-1} \otimes(x y)^{k-1}+(x y)^{k} \otimes x(y x)^{k-2}+(x y)^{k-1} \otimes x(y x)^{k-1}
\end{array}\right) ; \\
& \mathrm{T}^{0}\left(v_{2}^{\prime}\right)=(0, y \otimes 1), \quad \mathrm{T}^{1}\left(v_{2}^{\prime}\right)=\left(\begin{array}{c}
0 \\
y \otimes 1
\end{array}\right) \\
& \mathrm{T}^{2}\left(v_{2}^{\prime}\right)=\left(\begin{array}{c}
x \otimes x(y x)^{k-1}+y(x y)^{k-1} \otimes(y x)^{k-1}+(y x)^{k} \otimes y(x y)^{k-2}+(y x)^{k-1} \otimes y(x y)^{k-1} \\
\sum_{i=1}^{k}(x y)^{i} \otimes(x y)^{k-i}+\sum_{i=0}^{k-1}(y x)^{i} \otimes(y x)^{k-i}
\end{array}\right) ; \\
& \mathrm{T}^{0}(w)=(x \otimes y)^{*}, \\
& \mathrm{~T}^{1}(w)=\left(\begin{array}{c}
y \otimes(y x)^{k}-\sum_{i=1}^{k-1} i(x y)^{i+1} \otimes y(x y)^{k-1-i}-\sum_{i=1}^{k-2} i y(x y)^{i+1} \otimes(y x)^{k-1-i} \\
-\sum_{i=1}^{k-1} i(y x)^{i+1} \otimes x(y x)^{k-1-i}-\sum_{i=1}^{k-1} i x(y x)^{i} \otimes(x y)^{k-i}
\end{array}\right), \\
& \mathrm{T}^{2}(w)=\left(\begin{array}{cc}
-x \otimes x y x+y(x y)^{k-1} \otimes y x, & (x y)^{k-1} \otimes(y x)^{k}+(k-1)(x y)^{k} \otimes(y x)^{k-1} \\
(k-1)(y x)^{k} \otimes(x y)^{k-1} & 0
\end{array}\right), \\
& \mathrm{T}^{3}(w)=\left(\star, \quad-\sum_{i=2}^{k}(i-1)(x y)^{i} \otimes x(y x)^{k-i}-\sum_{i=1}^{k-1} i x(y x)^{i} \otimes(y x)^{k-i}\right)
\end{aligned}
$$

with

$$
\begin{aligned}
\mathrm{T}^{3}(w)_{1,1}= & \sum_{i=0}^{k-2}(k-1-i) y(x y)^{i} \otimes(x y)^{k-i}+\sum_{i=1}^{k-2}(k-1-i)(y x)^{i} \otimes y(x y)^{k-i} \\
& +y(x y)^{k-1} \otimes y x+(k-1) x(y x)^{k-1} \otimes x(y x)^{k-1}
\end{aligned}
$$

The proof of the lemma is a direct verification of the relations $\mu \mathrm{T}^{0}(b)=b, d_{i-1} \mathrm{~T}^{i}(b)=$ $\mathrm{T}^{i-1}(b) d_{i+\operatorname{deg} b}(0<i \leq \operatorname{deg} b)$, where $b \in \mathcal{Y}_{1} \backslash\{z\}$ with $\operatorname{deg} b>0$, and we leave it to the reader. 
Remark 4.1.3. For what follows, it should be noted that the formulas for the translates of the elements $u_{2}, v_{1}$, and $w$ remain valid if the field $K$ has an arbitrary characteristic.

Now the proof of Proposition 4.1.1 is completed with the help of calculations with the matrices presented in Lemma 4.1.2. We illustrate the corresponding techniques by proving the relation $u_{1} u_{2}=k p_{3}\left(u_{1}^{\prime}\right)^{2}$ (see (4.8)). The remaining relations are established similarly.

With the help of Lemma 4.1.2 we obtain $\mathrm{T}^{0}\left(u_{1}^{\prime}\right) \mathrm{T}^{1}\left(u_{1}^{\prime}\right)=\left(A_{1}, A_{2}\right)$, where

$$
\begin{aligned}
& A_{1}=y(x y)^{k-1} \otimes y(x y)^{k-3}+y(x y)^{k-2} \otimes y(x y)^{k-2}+\sum_{i=0}^{k-2} x(y x)^{i} \otimes(x y)^{k-2-i}, \\
& A_{2}=y(x y)^{k-2} \otimes x(y x)^{k-2}+1 \otimes 1+(y x)^{k-2} \otimes(y x)^{k-1}+(x y)^{k-2} \otimes(x y)^{k-1} .
\end{aligned}
$$

Consequently,

$$
\mu \cdot \mathrm{T}^{0}\left(u_{1}^{\prime}\right) \mathrm{T}^{1}\left(u_{1}^{\prime}\right)= \begin{cases}\left(x(y x)^{k-2}, 1\right) & \text { if } k>3, \\ \left(x y x, 1+(x y)^{3}\right) & \text { if } k=3 .\end{cases}
$$

In both cases the 2-cocycle $\mu \mathrm{T}^{0}\left(u_{1}^{\prime}\right) \mathrm{T}^{1}\left(u_{1}^{\prime}\right)$ is cohomological to the 2-cocycle $(0,1)$ (see Remark 3.1.3). On the other hand, using Lemma 4.1.2 again, we obtain

$\mathrm{T}^{0}\left(u_{1}\right) \mathrm{T}^{1}\left(u_{2}\right)=\left(x y x \otimes 1+\sum_{i=2}^{k-1}(i-1) y(x y)^{i} \otimes y(x y)^{k-1-i}, \sum_{i=2}^{k}(i-1)(x y)^{i} \otimes(y x)^{k-i}\right)$.

Thus, $\mu \mathrm{T}^{0}\left(u_{1}\right) \mathrm{T}^{1}\left(u_{2}\right)=\left(x y x,(k-1)(x y)^{k}\right)$. This 2-cocycle is cohomological to the cocycle $\left(0, k(x y)^{k}\right)$. Consequently, in $\mathrm{HH}^{*}(R)$ we have $u_{1} u_{2}=k p_{3}\left(u_{1}^{\prime}\right)^{2}$.

Proposition 4.1.4. Assume that $p=2$ and $k \geq 3$. The set $\mathcal{Y}_{1}$ defined in (4.1) generates $\mathrm{HH}^{*}(R)$ as a $K$-algebra.

Proof. Let $\mathcal{H}$ denote the $K$-subalgebra of $\operatorname{HH}^{*}(R)$ generated by the set $\mathcal{Y}_{1} \cup\{1\}$ (here 1 denotes the unity of the algebra $\left.\mathrm{HH}^{*}(R)\right)$. Since multiplication by $z$ gives an isomorphism $\mathrm{HH}^{i}(R) \rightarrow \mathrm{HH}^{i+4}(R)(i \geq 0)$, it suffices to prove that $\operatorname{HH}^{i}(R) \subset \mathcal{H}$ for $i \leq 3$.

By Remark 3.0.2, we have $\operatorname{HH}^{0}(R) \subset \mathcal{H}$. The following relations are satisfied in $\mathrm{HH}^{*}(R)$ :

$$
\begin{aligned}
& \left(x(y x)^{i}, 0\right)=p_{1}^{i-1} u_{2} \quad \text { with } 2 \leq i \leq k-2 ; \\
& \left(y(x y)^{k-1}, 0\right)=p_{2}^{\prime} u_{1}, \quad\left(0, x(y x)^{k-1}\right)=p_{2} u_{1}^{\prime}, \\
& \left((x y)^{k}, 0\right)=p_{3} u_{1}, \quad\left(0,(x y)^{k}\right)=p_{3} u_{1}^{\prime} .
\end{aligned}
$$

Now, Proposition 3.1.1 implies $\operatorname{HH}^{1}(R) \subset \mathcal{H}$.

Next, in the proof of Proposition 4.1.1 we saw that $(0,1)=\left(u_{1}^{\prime}\right)^{2}$; by symmetry we also have $(1,0)=u_{1}^{2}$. Moreover, in $\operatorname{HH}^{*}(R)$ we have $\left(y(x y)^{i}, x(y x)^{i}\right)=p_{1}^{i} v_{1}$ with $1 \leq i \leq k-2$. Using Proposition 3.2.3, a), we see that $\mathrm{HH}^{2}(R) \subset \mathcal{H}$.

As in the proof of Proposition 4.1.1, it is established that for the elements $1, x, y \in$ $Z^{3}(R)$, the following relations are satisfied in the algebra $\operatorname{HH}^{*}(R)$ :

$$
1=u_{1}^{3}, \quad x=u_{1}^{\prime} v_{1}, \quad y=u_{1} v_{1} .
$$

Since in $\operatorname{HH}^{*}(R)$ we also have $(x y)^{i}=p_{1}^{i-1} w$ for $1 \leq i \leq k-1$, Proposition 3.3 .2 implies the inclusion $\mathrm{HH}^{3}(R) \subset \mathcal{H}$.

Let $\mathcal{A}_{1}=K\left[\mathcal{X}_{1}\right] / I_{1}$ be the graded $K$-algebra defined in $\S 1$, with $\mathcal{X}_{1}$ as in (1.2), $I_{1}$ being the corresponding ideal of relations (see (1.4)-(1.15)). The (nonzero) images of monomials in $K\left[\mathcal{X}_{1}\right]$ under the canonical epimorphism $K\left[\mathcal{X}_{1}\right] \rightarrow \mathcal{A}_{1}$ are also called monomials. Any element $a \in \mathcal{A}_{1}$ is represented as a linear combination of monomials (with coefficients 
in $K$ ). Propositions 4.1.1 and 4.1 .4 imply that there exists a surjective homomorphism $\varphi: \mathcal{A}_{1} \rightarrow \mathrm{HH}^{*}(R)$ of graded $K$-algebras that takes the generators belonging to the set $\mathcal{X}_{1}$ to the corresponding generators in $\mathcal{Y}_{1}$ (see (4.1)). Note that no ambiguity is caused by employing the same letter to denote elements of both sets that correspond to each other. Let $\mathcal{A}_{1}=\bigoplus_{m \geq 0} \mathcal{A}_{1}^{m}$ be the direct decomposition of the algebra $\mathcal{A}_{1}$ into homogeneous direct summands. Now, part 1 a) of Theorem 1.1 is a consequence of the following statement.

Proposition 4.1.5. For any $m \geq 0$, we have

$$
\operatorname{dim}_{K} \mathcal{A}_{1}^{m}=\operatorname{dim}_{K} \mathrm{HH}^{m}(R) .
$$

Before proving this proposition, we state the following auxiliary fact.

Lemma 4.1.6. The following relations are satisfied in the algebra $\mathcal{A}_{1}$ :

$$
\begin{gathered}
p_{1}^{2} u_{1}=p_{1}^{2} u_{1}^{\prime}=0, \\
p_{1} u_{1}^{2}=p_{2} u_{1}^{2}=p_{2}^{\prime} u_{1}^{2}=p_{1}\left(u_{1}^{\prime}\right)^{2}=p_{2}\left(u_{1}^{\prime}\right)^{2}=p_{2}^{\prime}\left(u_{1}^{\prime}\right)^{2}=0, \\
u_{1}^{2} u_{1}^{\prime}=u_{1}\left(u_{1}^{\prime}\right)^{2}=u_{1}^{2} v_{1}=\left(u_{1}^{\prime}\right)^{2} v_{1}=u_{1}^{2} v_{2}=\left(u_{1}^{\prime}\right)^{2} v_{2}=u_{1}^{4}=\left(u_{1}^{\prime}\right)^{4}=0, \\
p_{1} u_{1} v_{1}=p_{1} u_{1}^{\prime} v_{1}=p_{2}^{\prime} u_{1} v_{1}=0, \quad p_{3} u_{1}^{3}=p_{1}^{k-1} w .
\end{gathered}
$$

Proof. To prove the lemma, it suffices to deduce the above relations directly from the defining relations of the algebra $\mathcal{A}_{1}$, and we leave this to the reader.

Proof of Proposition 4.1.5. On the polynomial ring $K\left[\mathcal{X}_{1}\right]$, where $\mathcal{X}_{1}$ is as in (1.2), we introduce a lexicographic order such that

$$
v_{2}^{\prime}>v_{2}>u_{2}>u_{1}^{\prime}>v_{1}>u_{1}>w>z>p_{1}>p_{2}>p_{2}^{\prime}>p_{3} .
$$

Let

$$
f=\left(v_{2}^{\prime}\right)^{\alpha^{\prime}} v_{2}^{\alpha} u_{2}^{\beta}\left(u_{1}^{\prime}\right)^{m^{\prime}} v_{1}^{\gamma} u_{1}^{m} w^{\varepsilon} z^{n} p_{1}^{t} p_{2}^{\zeta}\left(p_{2}^{\prime}\right)^{\zeta^{\prime}} p_{3}^{\eta}
$$

be a nonzero monomial in $\mathcal{A}_{1}$. The defining relations of the algebra $\mathcal{A}_{1}$ imply (see, in particular, Lemma 4.1.6) that in (4.11) we necessarily have $\alpha, \alpha^{\prime}, \beta, \varepsilon, \zeta, \zeta^{\prime}, \eta \in\{0,1\}$, $m \leq 3, m^{\prime} \leq 3$ and $t \leq k-1$. There are several additional restrictions on the occurrence of generators in (4.11) (e.g., we have at most one nonzero number among $\left\{t, \zeta, \zeta^{\prime}, \eta\right\}$ ); we shall analyze such restrictions below.

By definition, the reduction of a monomial $f$ in $\mathcal{A}_{1}$ is the process of replacement of some submonomials of $f$ by other elements of $\mathcal{A}_{1}$ in accordance with the following rules ( $a \mapsto b$ means the replacement of every entrance of the monomial $a$ by the element $b$ ):

$$
\begin{cases}v_{1} w \mapsto p_{1} u_{2} z, & v_{1}^{2} \mapsto p_{1}^{2} z, \\ u_{1} v_{2}^{\prime} \mapsto u_{1}^{\prime} v_{2} \mapsto p_{1}^{k-2} w, & p_{3} u_{1}^{3} \mapsto p_{1}^{k-1} w, \\ u_{2} v_{1} \mapsto p_{1} w, & u_{1} v_{2} \mapsto u_{1}^{\prime} v_{1}, \\ u_{1}^{\prime} v_{2}^{\prime} \mapsto u_{1} v_{1}, & u_{1} u_{1}^{\prime} \mapsto p_{1}^{k-2} v_{1} \quad \text { (if } k \text { odd), } \\ p_{1} v_{2}^{\prime} \mapsto p_{2}^{\prime} v_{2} \mapsto p_{2} v_{1} \mapsto p_{3} u_{1}^{2}, & p_{2} v_{2}^{\prime} \mapsto p_{1} v_{2} \mapsto p_{3}\left(u_{1}^{\prime}\right)^{2} \mapsto p_{2}^{\prime} v_{1}, \\ u_{1} u_{2} \mapsto p_{3}\left(u_{1}^{\prime}\right)^{2} \quad \text { (if } k \text { odd), } & u_{1}^{\prime} u_{2} \mapsto p_{3} u_{1}^{2} \quad \text { (if } k \text { odd), } \\ \left(u_{1}^{\prime}\right)^{3} \mapsto u_{1}^{3}, & p_{1}^{k-2} u_{2} \mapsto p_{2}^{\prime} u_{1}^{\prime} \mapsto p_{2} u_{1}, \\ p_{1} u_{1}^{\prime} \mapsto p_{2}^{\prime} u_{1}, & p_{2} u_{1}^{\prime} \mapsto p_{1} u_{1} .\end{cases}
$$

Any replacement on the above list is called an elementary step of reduction. Under such an elementary step, any nonzero monomial turns into a strictly smaller monomial with respect to the lexicographic order. Hence, after finitely many steps, we obtain a monomial to which we cannot apply any elementary step of reduction. We say that a presentation of an element $a \in \mathcal{A}_{1}$ as a linear combination of monomials has a normal form if reduction cannot be applied to any of these monomials. Since these elementary 
steps correspond to some relations satisfied in the algebra $\mathcal{A}_{1}$ (see (1.4)-(1.15) and Lemma 4.1.6), any element $a \in \mathcal{A}_{1}$ admits at least one presentation in the normal form.

Put $q_{i}=\operatorname{dim}_{K} \mathcal{A}_{1}^{i}$. We denote the number of monomials in $\mathcal{A}_{1}^{i}$ presented in the normal form by $\widetilde{q}_{i}$. It is clear that $\widetilde{q}_{i} \geq q_{i}$. Since there is an epimorphism $\mathcal{A}_{1}^{i} \rightarrow \mathrm{HH}^{i}(R)$, we see that $q_{i} \geq \operatorname{dim}_{K} \mathrm{HH}^{i}(R)$; consequently, it suffices to show that

$$
\widetilde{q}_{i}=\operatorname{dim}_{K} \mathrm{HH}^{i}(R) .
$$

We prove that all (nonzero) monomials having normal form are contained in the following list:

\begin{tabular}{|c|c|c|}
\hline Degree & Normal forms & Number \\
\hline \hline $4 n$ & $z^{n} p_{1}^{i} \quad(0 \leq i \leq k-1)$, & $k+3$ \\
\hline$z^{n} p_{2}, z^{n} p_{2}^{\prime}, z^{n} p_{3}$ & $k+5$ \\
\hline $4 n+1$ & $u_{2} z^{n} p_{1}^{i} \quad(0 \leq i \leq k-3)$, & $k+5$ \\
\hline $4 n+2$ & $u_{1} z^{n}, u_{1} z^{n} p_{1}, u_{1} z^{n} p_{2}, u_{1} z^{n} p_{2}^{\prime}, u_{1} z^{n} p_{3}, u_{1}^{\prime} z^{n}, u_{1}^{\prime} z^{n} p_{3}$ & \\
\hline $4 n+3$ & $v_{1} z^{n} p_{1}^{i} \quad(0 \leq i \leq k-2)$, & $k$ \\
\hline & $v_{1} z^{n} p_{2}^{\prime}, v_{2} z^{n}, v_{2}^{\prime} z^{n}, u_{1}^{2} z^{n}, u_{1}^{2} z^{n} p_{3},\left(u_{1}^{\prime}\right)^{2}$ & $k+3$ \\
\hline
\end{tabular}

It is easily seen that all monomials in this list have normal form.

Let $f$ be a nonzero monomial in (4.11) represented in the normal form. It is clear that $\gamma \leq 1$ (by the reduction $v_{1}^{2} \mapsto p_{1}^{2} z$ ) and $m^{\prime} \leq 2$ (by $\left(u_{1}^{\prime}\right)^{3} \mapsto u_{1}^{3}$ ). Next, we consider several cases successively.

1) Assume that the monomial $f$ contains a factor $w$. Some of the monomial relations satisfied in the algebra $\mathcal{A}_{1}$ (see (1.4)-(1.15) and Lemma 4.1.6) imply that $f$ does not contain $v_{1}, v_{2}, v_{2}^{\prime}, u_{1}, u_{1}^{\prime}, u_{2}, p_{2}, p_{2}^{\prime}, p_{3}$. Consequently, we have

$$
f=w z^{n} p_{1}^{i} \quad \text { for } 0 \leq i \leq k-1 .
$$

2) Assume that $f$ does not involve $w$, but involves $u_{1}$. Then $f$ cannot involve any one of the following elements: $u_{1}^{\prime}$ (because we have the reduction $u_{1} u_{1}^{\prime} \mapsto p_{1}^{k-1} v_{1}$ if $k$ is odd, and $u_{1} u_{1}^{\prime}=0$ if $k$ is even), $u_{2}$ (because we have the reduction $u_{1} u_{2} \mapsto p_{3}\left(u_{1}^{\prime}\right)^{2}$ if $k$ is odd, and $u_{1} u_{2}=0$ if $k$ is even), $v_{2}$ (because we have $u_{1} v_{2} \mapsto u_{1}^{\prime} v_{1}$ ), $v_{2}^{\prime}$ (because we have $\left.u_{1} v_{2}^{\prime} \mapsto p_{1}^{k-2} w\right)$. Consequently,

$$
f=v_{1}^{\gamma} u_{1}^{m} z^{n} p_{1}^{t} p_{2}^{\zeta}\left(p_{2}^{\prime}\right)^{\zeta^{\prime}} p_{3}^{\eta} .
$$

Here $t \leq 1$ (because $p_{1}^{2} u_{1}=0$; see Lemma 4.1.6). Moreover, if $m>1$, then $t=\zeta=\zeta^{\prime}=0$ by Lemma 4.1.6. if $m=3$, then also $\eta=0$; and if $\gamma=1$, then, by Lemma 4.1.6, $m=1$ and also $t=\zeta=\zeta^{\prime}=\eta=0$. Consequently, $f$ coincides with one of the following monomials:

$$
u_{1} z^{n}, u_{1} z^{n} p_{1}, u_{1} z^{n} p_{2}, u_{1} z^{n} p_{2}^{\prime}, u_{1} z^{n} p_{3}, u_{1}^{2} z^{n}, u_{1}^{2} z^{n} p_{3}, u_{1}^{3} z^{n}, v_{1} z^{n} u_{1} .
$$

3) Assume that $f$ involves neither $w$ nor $u_{1}$, and that $v_{1}$ occurs in $f$. Arguing as above, we see that $f$ does not involve $v_{2}, v_{2}^{\prime}, u_{2}, p_{2}, p_{3}$. Consequently,

$$
f=\left(u_{1}^{\prime}\right)^{m^{\prime}} v_{1} z^{n} p_{1}^{t}\left(p_{2}^{\prime}\right)^{\zeta^{\prime}}
$$

here $t \leq k-2$ and $m^{\prime} \leq 1$. If additionally we have $m^{\prime}=1$, then $t=\zeta^{\prime}=0$. Consequently, $f$ coincides with one of the following monomials:

$$
v_{1} z^{n} p_{1}^{i} \quad \text { with } 0 \leq i \leq k-2 ; \quad v_{1} z^{n} p_{2}^{\prime}, u_{1}^{\prime} v_{1} z^{n} .
$$


4) Assume that $w, u_{1}, v_{1}$ do not occur and $u_{1}^{\prime}$ occurs in $f$. Then it is easily seen that $f$ does not involve $v_{2}, v_{2}^{\prime}, u_{2}, p_{1}, p_{2}, p_{2}^{\prime}$. Consequently, $f=\left(u_{1}^{\prime}\right)^{m^{\prime}} z^{n} p_{3}^{\eta}$, and if $m^{\prime}>1$, then $\eta=0$. Hence, $f$ is one of the monomials

$$
u_{1}^{\prime} z^{n}, u_{1}^{\prime} z^{n} p_{3},\left(u_{1}^{\prime}\right)^{2} z^{n} .
$$

5) Assume that $w, u_{1}, v_{1}, u_{1}^{\prime}$ do not occur and $u_{2}$ occurs in $f$. Then $f$ does not involve $v_{2}, v_{2}^{\prime}, p_{2}, p_{2}^{\prime}, p_{3}$. Consequently, $f=u_{2} p_{1}^{i}$ for $i \leq k-3$, because we have the reduction $p_{1}^{k-2} u_{2} \mapsto p_{2} u_{1}$.

6) Assume that $w, u_{1}, v_{1}, u_{1}^{\prime}, u_{2}$ do not occur and $v_{2}$ occurs in $f$. Then $f$ does not involve $v_{2}^{\prime}, p_{1}, p_{2}, p_{2}^{\prime}, p_{3}$. Consequently, $f=v_{2} z^{n}$.

7) Assume that $w, u_{1}, v_{1}, u_{1}^{\prime}, u_{2}, v_{2}$ do not occur and $v_{2}^{\prime}$ occurs in $f$. Then $f$ does not involve $p_{1}, p_{2}, p_{2}^{\prime}, p_{3}$. Consequently, $f=v_{2}^{\prime} z^{n}$.

8) Finally, assume that $w, u_{1}, v_{1}, u_{1}^{\prime}, u_{2}, v_{2}, v_{2}^{\prime}$ do not occur in $f$. In this case, clearly, $f$ coincides with one of the monomials

$$
z^{n} p_{1}^{i} \quad \text { for } 0 \leq i \leq k-1 ; \quad z^{n} p_{2}, z^{n} p_{2}^{\prime}, z^{n} p_{3} .
$$

Consequently, we have shown that any monomial having the normal form is on the list above, and this completes the proof of (4.13).

Case 2: $k=2$. Consider the following homogeneous elements of the algebra $\mathrm{HH}^{*}(R)$ :

$$
\begin{array}{ll}
\text { of degree } 1: & u_{1}:=(1+x y, x), u_{1}^{\prime}:=(y, 1+y x) ; \\
\text { of degree 2: } & v_{1}:=(y, x), v_{2}:=(x, 0), v_{2}^{\prime}:=(0, y) ; \\
\text { of degree } 4: & z:=1 .
\end{array}
$$

Proposition 4.1.7. Assume that $p=2$ and $k=2$. Then, in the algebra $\mathrm{HH}^{*}(R)$, the elements of the set

$$
\mathcal{Y}_{2}=\left\{p_{1}, p_{2}, p_{2}^{\prime}, p_{3}, u_{1}, u_{1}^{\prime}, v_{1}, v_{2}, v_{2}^{\prime}, z\right\}
$$

satisfy relations (4.2), (4.4), (4.6), (4.7), (4.9), and (4.10), and also the relations

$$
p_{2} u_{1}=p_{2}^{\prime} u_{1}^{\prime}, u_{1}^{\prime} v_{2}=u_{1} v_{2}^{\prime}, v_{1}^{2}=0 \text {. }
$$

Proof. As in the proof of Proposition 4.1.1, we need to know the translates for the elements of the set $\mathcal{Y}_{2}$ that have positive degree. It is easily seen that the formulas for the translates of $v_{1}, v_{2}, v_{2}^{\prime}$ presented in Lemma 4.1.2 remain valid for $k=2$. Hence, we must compute additionally the corresponding translates for the elements $u_{1}$ and $u_{1}^{\prime}$.

Lemma 4.1.8. For the role of the translates (up to first order inclusive) of the elements $u_{1}$ and $u_{1}^{\prime}$ we can take the following maps:

$$
\begin{array}{ll}
\mathrm{T}^{0}\left(u_{1}\right)=((1+x y) \otimes 1, \quad x \otimes 1), & \\
\mathrm{T}^{1}\left(u_{1}\right)=\left(\begin{array}{cc}
1 \otimes(1+x y+y x) & (y+y x y) \otimes 1 \\
1 \otimes y+x \otimes x+x^{2} \otimes 1 & x \otimes 1+1 \otimes x+x \otimes y x
\end{array}\right) ; \\
\mathrm{T}^{0}\left(u_{1}^{\prime}\right)=\left(\begin{array}{cc}
y \otimes 1, \quad(1+y x) \otimes 1
\end{array}\right), \\
\mathrm{T}^{1}\left(u_{1}^{\prime}\right)=\left(\begin{array}{cc}
y \otimes 1+1 \otimes y+y \otimes x y & 1 \otimes x+y \otimes y+y^{2} \otimes 1 \\
(x+x y x) \otimes 1 & 1 \otimes(1+y x+x y)
\end{array}\right) .
\end{array}
$$

This lemma is proved by direct inspection (cf. Lemma 4.1.2).

Now, we can finish the proof of Proposition 4.1.7 with the help of the same technique as in the proof of Proposition 4.1.1.

Proposition 4.1.9. Assume that $p=2$ and $k=2$. The set $\mathcal{Y}_{2}$ defined in (4.14) generates $\mathrm{HH}^{*}(R)$ as a $K$-algebra. 
Proof. The proof is similar to that of Proposition 4.1.4. Additionally, it should be observed that the element $w:=x y \in Z^{3}(R)$ satisfies $w=u_{1}^{\prime} v_{2}$.

Let $\mathcal{A}_{2}=K\left[\mathcal{X}_{2}\right] / I_{2}$ be the graded $K$-algebra defined in $\S 1$ with $\mathcal{X}_{2}$ as in (1.16), $I_{2}$ being the corresponding ideal of relations. Propositions 4.1.7 and 4.1.9 imply that there exists a surjective homomorphism $\varphi: \mathcal{A}_{2} \rightarrow \mathrm{HH}^{*}(R)$ of graded $K$-algebras that takes the generators belonging to $\mathcal{X}_{2}$ to the corresponding generators in $\mathcal{Y}_{2}$. Let $\mathcal{A}_{2}=\bigoplus_{m \geq 0} \mathcal{A}_{2}^{m}$ be the direct decomposition into homogeneous direct summands. Now, part $1 \mathrm{~b}$ ) of Theorem 1.1 is a consequence of the following statement.

Proposition 4.1.10. For any $m \geq 0$, we have

$$
\operatorname{dim}_{K} \mathcal{A}_{2}^{m}=\operatorname{dim}_{K} \mathrm{HH}^{m}(R) .
$$

Proof. The proof of this proposition repeats the proof of Proposition 4.1.5 with minor changes. We only remark that on $K\left[\mathcal{X}_{2}\right]$ we introduce the lexicographic order such that

$$
v_{2}^{\prime}>v_{2}>u_{1}^{\prime}>v_{1}>u_{1}>z>p_{1}>p_{2}>p_{2}^{\prime}>p_{3} .
$$

Moreover, from the list (4.12) we remove the elementary steps of reduction in which $u_{2}$ or $w$ occurs.

4.2. char $K=3$. In this subsection, we assume that the ground field $K$ has characteristic three, if not stipulated otherwise.

Case 1: 3 does not divide $k$. Consider the following homogeneous elements in $\operatorname{HH}^{*}(R)$ :

$$
\begin{array}{ll}
\text { of degree } 0: & p_{1}, p_{2}, p_{2}^{\prime} \quad(\text { see }(3.4)) ; \\
\text { of degree 1: } & u_{2}:=(x y x, 0), u_{3}:=(x,-y) ; \\
\text { of degree 2: } & v_{0}:=(1,0), v_{0}^{\prime}:=(0,1), v_{1}:=(y, x) ; \\
\text { of degree } 3: & w:=x y ; \\
\text { of degree } 4: & z:=1 .
\end{array}
$$

Proposition 4.2.1. Assume that $p=3$ and 3 does not divide $k$. Then, in the algebra $\mathrm{HH}^{*}(R)$, the elements of the set

$$
\mathcal{Y}_{3}=\left\{p_{1}, p_{2}, p_{2}^{\prime}, u_{2}, u_{3}, v_{0}, v_{0}^{\prime}, v_{1}, w, z\right\}
$$

satisfy the following relations:

$$
\begin{gathered}
p_{1}^{k+1}=p_{2}^{2}=\left(p_{2}^{\prime}\right)^{2}=p_{1} p_{2}=p_{1} p_{2}^{\prime}=p_{2} p_{2}^{\prime}=0 ; \\
p_{1} v_{0}=p_{1} v_{0}^{\prime}=p_{2} v_{0}=p_{2}^{\prime} v_{0}^{\prime}=p_{2} v_{1}=p_{2}^{\prime} v_{1}=p_{1}^{k-1} v_{1}=0 ; \\
p_{2} u_{2}=p_{2}^{\prime} u_{2}=p_{1}^{k-1} u_{2}=0 ; \\
p_{2} v_{0}^{\prime}+p_{2}^{\prime} v_{0}=0, \quad p_{1} u_{3}=u_{2} u_{3}=u_{3} v_{1}=0 ; \\
p_{2} w=p_{2}^{\prime} w=u_{2} v_{0}=u_{2} v_{0}^{\prime}=u_{2} w=p_{1}^{k} z=0, \quad u_{2} v_{1}=p_{1} w ; \\
p_{2}^{\prime} u_{3} v_{0}=p_{1}^{k-1} w, \quad u_{3} w=0 ; \\
v_{0} v_{0}^{\prime}=v_{0} v_{1}=v_{0}^{\prime} v_{1}=0, \quad v_{0}^{2}=2 p_{2}^{\prime} z, \quad\left(v_{0}^{\prime}\right)^{2}=2 p_{2} z, \quad v_{1}^{2}=-p_{1}^{2} z ; \\
v_{0} w=v_{0}^{\prime} w=0, \quad v_{1} w=-p_{1} u_{2} z .
\end{gathered}
$$

Proof. To prove these relations, we need to know the translates (of suitable orders) for the elements of $\mathcal{Y}_{3}$ that have positive degree. By Remark 4.1.3, we must calculate only the translates for $u_{3}, v_{0}, v_{0}^{\prime}$. 
Lemma 4.2.2. For the role of the translates of the elements $u_{3}, v_{0}$, and $v_{0}^{\prime}$, we can take the following maps:

$$
\begin{aligned}
& \mathrm{T}^{0}\left(u_{3}\right)=(x \otimes 1, \quad-y \otimes 1), \\
& \mathrm{T}^{1}\left(u_{3}\right)=\left(\begin{array}{cc}
-x \otimes 1-\sum_{i=0}^{k-2} y(x y)^{i} \otimes y(x y)^{k-2-i} & 0 \\
0 & y \otimes 1+\sum_{i=0}^{k-2} x(y x)^{i} \otimes x(y x)^{k-2-i}
\end{array}\right) ; \\
& \mathrm{T}^{0}\left(v_{0}\right)=(1 \otimes 1,0), \quad \mathrm{T}^{1}\left(v_{0}\right)=\left(\begin{array}{c}
1 \otimes 1 \\
0
\end{array}\right), \\
& \mathrm{T}^{2}\left(v_{0}\right)=\left(\begin{array}{c}
\sum_{i=0}^{k-1} y(x y)^{i} \otimes(y x)^{k-1-i}+\sum_{i=0}^{k-1}(x y)^{i} \otimes y(x y)^{k-1-i} \\
(y x)^{k-1} \otimes(x y)^{k-1}
\end{array}\right) ; \\
& \mathrm{T}^{0}\left(v_{0}^{\prime}\right)=(0,1 \otimes 1), \quad \mathrm{T}^{1}\left(v_{0}^{\prime}\right)=\left(\begin{array}{c}
0 \\
1 \otimes 1
\end{array}\right), \\
& \mathrm{T}^{2}\left(v_{0}^{\prime}\right)=\left(\begin{array}{c}
(x y)^{k-1} \otimes(y x)^{k-1} \\
\sum_{i=0}^{k-1} x(y x)^{i} \otimes(x y)^{k-1-i}+\sum_{i=0}^{k-1}(y x)^{i} \otimes x(y x)^{k-1-i}
\end{array}\right) .
\end{aligned}
$$

This lemma is proved by direct calculation (cf. Lemma 4.1.2).

Remark 4.2.3. It should be noted that the formulas for the translates of the elements $v_{0}$ and $v_{0}^{\prime}$ remain valid in the cases where $k$ is divisible by $p=3$ or $p \notin\{2,3\}$.

Now, the proof of Proposition 4.2.1 can be completed as was done in the proof of Proposition 4.1.1.

Proposition 4.2.4. Assume that $p=3$ and 3 does not divide $k$. The set $\mathcal{Y}_{3}$ defined in (4.16) generates $\mathrm{HH}^{*}(R)$ as a $K$-algebra.

Proof. The proof is similar to that of Proposition 4.1.4 and employs, in particular, Propositions 3.1.5, 3.2.3. b) and 3.3.2. Besides the relations obtained in the proof of Proposition 4.1.4 the following relations should be used:

$$
\begin{array}{ll}
\text { in } \mathrm{HH}^{1}(R): & \left((x y)^{k}, 0\right)=p_{2}^{\prime} u_{3}, \quad\left(0,(x y)^{k}\right)=-p_{2} u_{3} ; \\
\text { in } \mathrm{HH}^{2}(R): & \left(y(x y)^{k-1}, 0\right)=p_{2}^{\prime} v_{0} ; \\
\text { in } \mathrm{HH}^{3}(R): & x=u_{3} v_{0}, \quad y=-u_{3} v_{0}^{\prime} ; \\
\text { in } \mathrm{HH}^{4}(R): & (x y)^{i}+(y x)^{i}=p_{1}^{i} z(1 \leq i \leq k-1), \quad x(y x)^{k-1}=p_{2} z, y(x y)^{k-1}=p_{1}^{\prime} z .
\end{array}
$$

Let $\mathcal{A}_{3}=K\left\langle\mathcal{X}_{3}\right\rangle / I_{3}$ be the graded $K$-algebra defined in $\S 1$, with $\mathcal{X}_{3}$ as in (1.17), $I_{3}$ being the corresponding ideal of relations (see (1.19)-(1.31)). Propositions 4.2.1 and 4.2.4 imply that there exists a surjective homomorphism $\varphi: \mathcal{A}_{3} \rightarrow \operatorname{HH}^{*}(R)$ of graded $K$ algebras that takes the generators belonging to $\mathcal{X}_{3}$ to the corresponding generators in $\mathcal{Y}_{3}$ (see (4.16)). Let $\mathcal{A}_{3}=\bigoplus_{m>0} \mathcal{A}_{3}^{m}$ be the direct decomposition into homogeneous direct summands. Now, part 2 a) of Theorem 1.1 is a consequence of the following statement.

Proposition 4.2.5. For any $m \geq 0$, we have

$$
\operatorname{dim}_{K} \mathcal{A}_{3}^{m}=\operatorname{dim}_{K} \operatorname{HH}^{m}(R) .
$$

Proof. On the polynomial ring $K\left[\mathcal{X}_{3}\right]$, we introduce the lexicographic order such that

$$
v_{0}^{\prime}>v_{0}>v_{1}>u_{2}>u_{3}>w>z>p_{1}>p_{2}>p_{2}^{\prime} .
$$

Since the algebra $\mathcal{A}_{3}$ is graded-commutative, any nonzero monomial $f \in \mathcal{A}_{3}$ is represented, up to a scalar factor, in the form

$$
f=\left(v_{0}^{\prime}\right)^{\alpha^{\prime}} v_{0}^{\alpha} v_{1}^{\beta} u_{2}^{\gamma} u_{3}^{\varepsilon} w^{\zeta} z^{n} p_{1}^{t} p_{2}^{\theta}\left(p_{2}^{\prime}\right)^{\theta^{\prime}} .
$$


Such representations of elements of the algebra $\mathcal{A}_{3}$ can be viewed as elements of $K\left[\mathcal{X}_{3}\right]$. Consider the following list of elementary steps of reduction:

$$
\begin{cases}v_{1} w \mapsto p_{1} u_{2} z, & u_{2} v_{1} \mapsto p_{1} w, \\ p_{2} v_{0}^{\prime} u_{3} \mapsto p_{2}^{\prime} v_{0} u_{3} \mapsto p_{1}^{k-1} w, & v_{1}^{2} \mapsto p_{1}^{2} z, \\ v_{0}^{2} \mapsto p_{2}^{\prime} z, & \left(v_{0}^{\prime}\right)^{2} \mapsto p_{2} z, \\ p_{2} v_{0}^{\prime} \mapsto p_{2}^{\prime} v_{0} . & \end{cases}
$$

As in the proof of Proposition 4.1.5, we can define the normal form of an element $a \in \mathcal{A}_{3}$ (with respect to the steps of reduction (4.24)) and then verify that any element $a \in \mathcal{A}_{3}$ admits at least one normal form. Finally, studying several cases successively, we prove that all (nonzero) monomials having normal form are on the following list:

\begin{tabular}{|c|c|c|}
\hline Degree & Normal forms & Number \\
\hline 0 & $\begin{array}{c}p_{1}^{i}(1 \leq i \leq k), \\
1, p_{2}, p_{2}^{\prime}\end{array}$ & $k+3$ \\
\hline $4 n, n>0$ & $\begin{array}{c}z^{n} p_{1}^{i}(0 \leq i \leq k-1), \\
z^{n} p_{2}, z^{n} p_{2}^{\prime}\end{array}$ & $k+2$ \\
\hline $4 n+1$ & $\begin{array}{c}u_{2} z^{n} p_{1}^{i}(0 \leq i \leq k-2) \\
u_{3} z^{n}, u_{3} z^{n} p_{2}, u_{3} z^{n} p_{2}^{\prime}\end{array}$ & $k+2$ \\
\hline $4 n+2$ & $\begin{array}{c}v_{1} z^{n} p_{1}^{i}(0 \leq i \leq k-2) \\
v_{0} z^{n}, v_{0} z^{n} p_{2}^{\prime}, v_{0}^{\prime} z^{n}\end{array}$ & $k+2$ \\
\hline $4 n+3$ & $\begin{array}{c}w z^{n} p_{1}^{i}(0 \leq i \leq k-1) \\
v_{0} u_{3} z^{n}, v_{0}^{\prime} u_{3} z^{n}\end{array}$ & $k+2$ \\
\hline
\end{tabular}

As in the proof of Proposition 4.1.5, this implies (4.23). The details are left to the reader.

Case 2: 3 divides $k$. Consider the following homogeneous elements of $\mathrm{HH}^{*}(R)$ :

$$
\begin{array}{lll}
\text { of degree } 0: & p_{1}, p_{2}, p_{2}^{\prime} \quad(\text { see }(3.4)) ; \\
\text { of degree } 1: & \widetilde{u}_{1}:=(x, 0), \widetilde{u}_{1}^{\prime}:=(0, y) ; \\
\text { of degree } 2: & v_{0}, v_{0}^{\prime}, v_{1} \quad(\text { see (4.15) }) ; \\
\text { of degree } 3: & \widetilde{w}:=1 ; \\
\text { of degree } 4: & z:=1 .
\end{array}
$$

Proposition 4.2.6. Assume that $p=3$ and 3 divides $k$. Then, in the algebra $\mathrm{HH}^{*}(R)$, the elements of the set

$$
\mathcal{Y}_{4}=\left\{p_{1}, p_{2}, p_{2}^{\prime}, \widetilde{u}_{1}, \widetilde{u}_{1}^{\prime}, v_{0}, v_{0}^{\prime}, v_{1}, \widetilde{w}, z\right\}
$$

satisfy relations (4.17), (4.18), and (4.21), and the relations

$$
\begin{gathered}
p_{2} \widetilde{u}_{1}=p_{2}^{\prime} \widetilde{u}_{1}^{\prime}=p_{1}^{k} \widetilde{u}_{1}=p_{1}^{k} \widetilde{u}_{1}^{\prime}=0, \quad p_{1} \widetilde{u}_{1}=p_{1} \widetilde{u}_{1}^{\prime} ; \\
\widetilde{u}_{1} \widetilde{u}_{1}^{\prime}=0, \quad p_{1}^{k-1} v_{1}=p_{2}^{\prime} v_{0}+p_{2} v_{0}^{\prime} ; \\
p_{2} \widetilde{w}=p_{2}^{\prime} \widetilde{w}=\widetilde{u}_{1}^{\prime} v_{0}=\widetilde{u}_{1} v_{0}^{\prime}=0, \quad \widetilde{u}_{1}^{\prime} v_{1}=\widetilde{u}_{1} v_{1}, \quad p_{1} \widetilde{w}=2 \widetilde{u}_{1} v_{1} ; \\
\widetilde{u}_{1} \widetilde{w}=\widetilde{u}_{1}^{\prime} \widetilde{w}=v_{0} \widetilde{w}=v_{0}^{\prime} \widetilde{w}=0, \quad v_{1} \widetilde{w}=-2 u_{2} z .
\end{gathered}
$$

Proof. By Remark 4.2.3, to prove (4.26) it remains to calculate the translates of the elements $\widetilde{u}_{1}, \widetilde{u}_{1}^{\prime}$, and $\widetilde{w}$. 
Lemma 4.2.7. For the role of the translates of $\widetilde{u}_{1}, \widetilde{u}_{1}^{\prime}$, and $\widetilde{w}$, we can take the following maps:

$$
\begin{aligned}
& \mathrm{T}^{0}\left(\widetilde{u}_{1}\right)=(x \otimes 1, \quad 0), \\
& \mathrm{T}^{1}\left(\widetilde{u}_{1}\right)=\left(\begin{array}{cc}
-x \otimes 1+\sum_{i=1}^{k-2} i y(x y)^{i} \otimes y(x y)^{k-2-i} & \sum_{i=1}^{k-1} i(x y)^{i} \otimes(y x)^{k-1-i} \\
\sum_{i=1}^{k-1} i(y x)^{i} \otimes(x y)^{k-1-i} & \sum_{i=0}^{k-2}(i+1) x(y x)^{i} \otimes x(y x)^{k-2-i}
\end{array}\right) ; \\
& \mathrm{T}^{0}\left(\widetilde{u}_{1}^{\prime}\right)=(0, \quad y \otimes 1), \\
& \mathrm{T}^{1}\left(\widetilde{u}_{1}^{\prime}\right)=\left(\begin{array}{cc}
\sum_{i=0}^{k-2}(i+1) y(x y)^{i} \otimes y(x y)^{k-2-i} & \sum_{i=1}^{k-1} i(x y)^{i} \otimes(y x)^{k-1-i} \\
\sum_{i=1}^{k-1} i(y x)^{i} \otimes(x y)^{k-1-i} & -y \otimes 1+\sum_{i=1}^{k-2} i x(y x)^{i} \otimes x(y x)^{k-2-i}
\end{array}\right) ; \\
& \mathrm{T}^{0}(\widetilde{w})=(1 \otimes 1)^{*}, \\
& \mathrm{~T}^{1}(\widetilde{w})=\left(\begin{array}{c}
-\sum_{i=0}^{k-1}(2 i+1) y(x y)^{i} \otimes(y x)^{k-1-i}-\sum_{i=0}^{k-1}(2 i+1)(x y)^{i} \otimes y(x y)^{k-1-i} \\
-\sum_{i=1}^{k-1} 2 i(y x)^{i} \otimes x(y x)^{k-1-i}-\sum_{i=0}^{k-1}(2 i+2) x(y x)^{i} \otimes(x y)^{k-1-i}
\end{array}\right), \\
& \mathrm{T}^{2}(\widetilde{w})=\left(\begin{array}{cc}
-2 x \otimes x+x^{2} \otimes 1+1 \otimes x^{2} & -(x y)^{k-1} \otimes(y x)^{k-1} \\
-(y x)^{k-1} \otimes(x y)^{k-1} & 0
\end{array}\right), \\
& \mathrm{T}^{3}(\widetilde{w})=\left(-\sum_{i=0}^{k-1}(2 i+1)(y x)^{i} \otimes(x y)^{k-1-i} \cdot(y \otimes 1+1 \otimes y), \quad \star\right)
\end{aligned}
$$

with

$$
\mathrm{T}^{3}(\widetilde{w})_{1,2}=-2 \sum_{i=0}^{k-2}(i+1) x(y x)^{i} \otimes(y x)^{k-1-i}-\sum_{i=0}^{k-1} 2 i(x y)^{i} \otimes x(y x)^{k-1-i} .
$$

This lemma is proved by direct calculation (cf. Lemma 4.1.2).

Remark 4.2.8. Observe that the formulas for the translates of $\widetilde{w}$ remain valid in the case where $p \notin\{2,3\}$ and $p$ divides $k$.

Now, the proof of Proposition 4.2.6 can be completed as was done in the proof of Proposition 4.1.1.

Proposition 4.2.9. Assume that $p=3$ and 3 divides $k$. The set $\mathcal{Y}_{4}$ defined in (4.25) generates $\mathrm{HH}^{*}(R)$ as a $K$-algebra.

Proof. The proof is similar to that of Proposition 4.1.4 and employs, in particular, Propositions $3.1 .8,3.2 .3 \mathrm{~b}$ ), and 3.3 .2

Let $\mathcal{A}_{4}=K\left\langle\mathcal{X}_{4}\right\rangle / I_{4}$ be the graded $K$-algebra defined in $\S 1$, with $\mathcal{X}_{4}$ as in (1.32), $I_{4}$ being the corresponding ideal of relations. Propositions 4.2.6 and 4.2 .9 imply that there exists a surjective homomorphism $\varphi: \mathcal{A}_{4} \rightarrow \mathrm{HH}^{*}(R)$ of graded $K$-algebras that takes the generators belonging to $\mathcal{X}_{4}$ to the corresponding generators in $\mathcal{Y}_{4}$ (see (4.25)). Let $\mathcal{A}_{4}=\bigoplus_{m \geq 0} \mathcal{A}_{4}^{m}$ be the direct decomposition into homogeneous direct summands. Now, part $2 \mathrm{~b}$ ) of Theorem 1.1 is a consequence of the following statement.

Proposition 4.2.10. For any $m \geq 0$, we have

$$
\operatorname{dim}_{K} \mathcal{A}_{4}^{m}=\operatorname{dim}_{K} \mathrm{HH}^{m}(R) .
$$

Proof. The proof repeats that of Proposition 4.2.5. On the polynomial ring $K\left[\mathcal{X}_{4}\right]$, we introduce the lexicographic order such that

$$
v_{1}>v_{0}^{\prime}>v_{0}>\widetilde{u}_{1}^{\prime}>\widetilde{u}_{1}>\widetilde{w}>z>p_{1}>p_{2}>p_{2}^{\prime} .
$$


Then we consider the following list of elementary steps of reduction:

$$
\begin{array}{ll}
\widetilde{u}_{1}^{\prime} v_{1} \mapsto \widetilde{u}_{1} v_{1} \mapsto p_{1} \widetilde{w}, & p_{2} \widetilde{u}_{1}^{\prime} v_{0}^{\prime} \mapsto p_{2}^{\prime} \widetilde{u}_{1} v_{0} \mapsto p_{1}^{k} \widetilde{w}, \\
p_{1}^{k-1} v_{1} \mapsto p_{2}^{\prime} v_{0}+p_{2} v_{0}^{\prime}, & v_{1}^{2} \mapsto p_{1}^{2} z, \\
v_{0}^{2} \mapsto p_{2}^{\prime} z, & \left(v_{0}^{\prime}\right)^{2} \mapsto p_{2} z, \\
p_{1} \widetilde{u}_{1}^{\prime} \mapsto p_{1} \widetilde{u}_{1} . &
\end{array}
$$

This list includes the steps $p_{2} \widetilde{u}_{1}^{\prime} v_{0}^{\prime} \mapsto p_{2}^{\prime} \widetilde{u}_{1} v_{0} \mapsto p_{1}^{k} \widetilde{w}$, because the defining relations of the algebra $\mathcal{A}_{4}$ imply that $p_{1}^{k} \widetilde{w}=2 p_{2} \widetilde{u}_{1}^{\prime} v_{0}^{\prime}=2 p_{2}^{\prime} \widetilde{u}_{1} v_{0}$. Finally, it can be checked that all (nonzero) monomials having normal form are contained in the following list:

\begin{tabular}{|c|c|c|}
\hline Degree & Normal forms & Number \\
\hline \hline $4 n$ & $\begin{array}{c}z^{n} p_{1}^{i}(0 \leq i \leq k), \\
z^{n} p_{2}, z^{n} p_{2}^{\prime}\end{array}$ & $k+3$ \\
\hline $4 n+1$ & $\begin{array}{c}\widetilde{u}_{1} z^{n} p_{1}^{i}(0 \leq i \leq k-1), \\
\widetilde{u}_{1} z^{n} p_{2}^{\prime}, \widetilde{u}_{1}^{\prime} z^{n}, \widetilde{u}_{1}^{\prime} z^{n} p_{2}\end{array}$ & $k+3$ \\
\hline $4 n+2$ & $\begin{array}{c}v_{1} z^{n} p_{1}^{i}(0 \leq i \leq k-2), \\
v_{0} z^{n}, v_{0} z^{n} p_{2}^{\prime}, v_{0}^{\prime} z^{n}, v_{0}^{\prime} z^{n} p_{2}\end{array}$ & $k+3$ \\
\hline $4 n+3$ & $\begin{array}{c}\widetilde{w} z^{n} p_{1}^{i}(0 \leq i \leq k), \\
v_{0} \widetilde{u}_{1}, v_{0}^{\prime} \widetilde{u}_{1}^{\prime}\end{array}$ & $k+3$ \\
\hline
\end{tabular}

This leads to (4.27).

4.3. char $K \notin\{2,3\}$. In this subsection, we assume that the ground field $K$ has characteristic different from 2 and 3.

Case 1: $p$ divides neither $3-2 k$ nor $k$. Consider the following homogeneous elements in $\operatorname{HH}^{*}(R)$ :

$$
\begin{array}{ll}
\text { of degree } 0: & p_{1}, p_{2}, p_{2}^{\prime} \quad(\text { see (3.4) }) ; \\
\text { of degree 1: } & u_{2}:=(x y x, 0), u_{4}:=\left((x y)^{k}, 0\right), u_{4}^{\prime}:=\left(0,(x y)^{k}\right) ; \\
\text { of degree 2: } & v_{0}, v_{0}^{\prime}, v_{1} \quad(\text { see (4.15) }) ; \\
\text { of degree 3: } & w_{1}:=x, w_{1}^{\prime}:=y, w:=x y ; \\
\text { of degree 4: } & z:=1 .
\end{array}
$$

Proposition 4.3.1. Assume that $p$ divides neither $3-2 k$ nor $k$. Then, in the algebra $\mathrm{HH}^{*}(R)$, the elements of the set

$$
\mathcal{Y}_{5}=\left\{p_{1}, p_{2}, p_{2}^{\prime}, u_{2}, u_{4}, u_{4}^{\prime}, v_{0}, v_{0}^{\prime}, v_{1}, w_{1}, w_{1}^{\prime}, w, z\right\}
$$

satisfy relations (4.17), (4.18), (4.19), (4.20), (4.21), and (4.22), and also the relations

$$
\begin{gathered}
p_{1} u_{4}=p_{1} u_{4}^{\prime}=p_{2} u_{4}=p_{2} u_{4}^{\prime}=p_{2}^{\prime} u_{4}=p_{2}^{\prime} u_{4}^{\prime}=0 ; \\
u_{2} u_{4}=u_{2} u_{4}^{\prime}=u_{4} u_{4}^{\prime}=0 ; \\
p_{1} w_{1}=p_{1} w_{1}^{\prime}=p_{2} w_{1}=p_{2}^{\prime} w_{1}^{\prime}=0 ; \\
p_{2} w_{1}^{\prime}=p_{2}^{\prime} w_{1}=u_{4} v_{0}=u_{4}^{\prime} v_{0}^{\prime} ; \\
u_{4} v_{0}^{\prime}=u_{4}^{\prime} v_{0}=u_{1} v_{1}=u_{1}^{\prime} v_{1}=0 ; \\
v_{0} w_{1}^{\prime}=v_{0}^{\prime} w_{1}=v_{1} w_{1}=v_{1} w_{1}^{\prime}=0, \quad v_{0} w_{1}=2 u_{4} z, \quad v_{0}^{\prime} w_{1}^{\prime}=2 u_{4}^{\prime} z ; \\
w_{1} w_{1}^{\prime}=0 ; \\
u_{4}^{\prime} v_{0}^{\prime}=p_{1}^{k-1} w, \quad u_{4} w=u_{4}^{\prime} w=w_{1} w=w_{1}^{\prime} w=0 .
\end{gathered}
$$

Proof. By Remarks 4.1.3 and 4.2.3. to prove the relations mentioned above it remains to calculate the translates (of suitable orders) of the elements $u_{4}, u_{4}^{\prime}, w_{1}$, and $w_{1}^{\prime}$. 
Lemma 4.3.2. For the role of the translates of $u_{4}, u_{4}^{\prime}, w_{1}$, and $w_{1}^{\prime}$, we can take the following maps:

$$
\begin{aligned}
& \mathrm{T}^{0}\left(u_{4}\right)=\left((x y)^{k} \otimes 1, \quad 0\right), \quad \mathrm{T}^{1}\left(u_{4}\right)=\left(\begin{array}{cc}
-(x y)^{k} \otimes 1 & 0 \\
0 & (x y)^{k} \otimes x(y x)^{k-2}
\end{array}\right) ; \\
& \mathrm{T}^{0}\left(u_{4}^{\prime}\right)=\left(\begin{array}{lc}
0, & (x y)^{k} \otimes 1
\end{array}\right), \quad \mathrm{T}^{1}\left(u_{4}^{\prime}\right)=\left(\begin{array}{cc}
(x y)^{k} \otimes y(x y)^{k-2} & 0 \\
0 & -(x y)^{k} \otimes 1
\end{array}\right) ; \\
& \mathrm{T}^{0}\left(w_{1}\right)=(x \otimes 1)^{*}, \\
& \mathrm{~T}^{1}\left(w_{1}\right)=\left(\begin{array}{c}
\sum_{i=1}^{k-1} y(x y)^{i-1} \otimes x(y x)^{k-i}+\sum_{i=0}^{k-1}(x y)^{i} \otimes(x y)^{k-i}-(x y)^{k} \otimes 1 \\
(y x)^{k-1} \otimes y(x y)^{k-1}
\end{array}\right), \\
& \mathrm{T}^{2}\left(w_{1}\right)=\left(\begin{array}{cc}
(x y)^{k} \otimes 1+1 \otimes(x y)^{k} & y(x y)^{k-1} \otimes y+(x y)^{k-1} \otimes x(y x)^{k-1} \\
x(y x)^{k-1} \otimes(x y)^{k-1} & 0
\end{array}\right), \\
& \mathrm{T}^{3}\left(w_{1}\right)=\left(\sum_{i=0}^{k-1} y(x y)^{i} \otimes x(y x)^{k-1-i}+\sum_{i=0}^{k-1}(y x)^{i} \otimes(y x)^{k-i}, \quad-2 y(x y)^{k-1} \otimes(y x)^{k-1}\right) ; \\
& \mathrm{T}^{0}\left(w_{1}^{\prime}\right)=(x \otimes 1)^{*}, \\
& \mathrm{~T}^{1}\left(w_{1}^{\prime}\right)=\left(\begin{array}{c}
(x y)^{k-1} \otimes x(y x)^{k-1} \\
\sum_{i=1}^{k-1} x(y x)^{i-1} \otimes y(x y)^{k-i}+\sum_{i=0}^{k-1}(y x)^{i} \otimes(y x)^{k-i}-(x y)^{k} \otimes 1
\end{array}\right), \\
& \mathrm{T}^{2}\left(w_{1}^{\prime}\right)=\left(\begin{array}{cc}
0 & y(x y)^{k-1} \otimes(y x)^{k-1} \\
x(y x)^{k-1} \otimes x+(y x)^{k-1} \otimes y(x y)^{k-1} & (x y)^{k} \otimes 1+1 \otimes(x y)^{k}
\end{array}\right), \\
& \mathrm{T}^{3}\left(w_{1}^{\prime}\right)=\left(-2 y(x y)^{k-1} \otimes(y x)^{k-1}, \quad \sum_{i=0}^{k-1} x(y x)^{i} \otimes y(x y)^{k-1-i}+\sum_{i=0}^{k-1}(x y)^{i} \otimes(x y)^{k-i}\right) .
\end{aligned}
$$

Using Lemma 4.3.2 we can complete the proof of Proposition 4.3.1 as was done in the proof of Proposition 4.1.1.

Proposition 4.3.3. Assume that $p$ divides neither $3-2 k$ nor $k$. The set $\mathcal{Y}_{5}$ defined in (4.29) generates $\mathrm{HH}^{*}(R)$ as a K-algebra.

The proof is similar to that of Proposition 4.1.4

Let $\mathcal{A}_{5}=K\left\langle\mathcal{X}_{5}\right\rangle / I_{5}$ be the graded $K$-algebra defined in $\S 1$, with $\mathcal{X}_{5}$ as in (1.33), $I_{5}$ being the corresponding ideal of relations. Propositions 4.3.1 and 4.3 .3 imply that there exists a surjective homomorphism $\varphi: \mathcal{A}_{5} \rightarrow \mathrm{HH}^{*}(R)$ of graded $K$-algebras that takes the generators belonging to $\mathcal{X}_{5}$ to the corresponding generators in $\mathcal{Y}_{5}$ (see (4.29)). Let $\mathcal{A}_{5}=\bigoplus_{m \geq 0} \mathcal{A}_{5}^{m}$ be the direct decomposition into homogeneous direct summands. Now, part $3 \mathrm{a}$ ) of Theorem 1.1 is a consequence of the following statement.

Proposition 4.3.4. For any $m \geq 0$, we have

$$
\operatorname{dim}_{K} \mathcal{A}_{5}^{m}=\operatorname{dim}_{K} \mathrm{HH}^{m}(R) .
$$

Proof. On $K\left[\mathcal{X}_{5}\right]$, we introduce the lexicographic order such that

$$
v_{0}^{\prime}>v_{0}>v_{1}>u_{2}>u_{4}^{\prime}>u_{4}>w>w_{1}^{\prime}>w_{1}>z>p_{1}>p_{2}>p_{2}^{\prime} .
$$


Consider the following list of elementary steps of reduction:

$$
\begin{cases}v_{0} w_{1} \mapsto u_{4} z, & v_{0}^{\prime} w_{1}^{\prime} \mapsto u_{4}^{\prime} z, \\ v_{0}^{2} \mapsto p_{2}^{\prime} z, & \left(v_{0}^{\prime}\right)^{2} \mapsto p_{2} z, \\ v_{1}^{2} \mapsto p_{1}^{2} z, & u_{2} v_{1} \mapsto p_{1} w, \\ u_{4}^{\prime} v_{0}^{\prime} \mapsto u_{4} v_{0} \mapsto p_{2}^{\prime} w_{1}, & p_{1}^{k-1} w \mapsto p_{2} w_{1}^{\prime} \mapsto p_{2}^{\prime} w_{1}, \\ v_{1} w \mapsto p_{1} u_{2} z . & \end{cases}
$$

As in the proof of Proposition 4.1.5, we can define the normal form of an element $a \in \mathcal{A}_{5}$ and then verify that any element $a \in \mathcal{A}_{5}$ admits at least one normal form (see also the proof of Proposition 4.2.5). After that, we check that all (nonzero) monomials having normal form are on the following list:

\begin{tabular}{|c|c|c|}
\hline Degree & Normal forms & Number \\
\hline \hline 0 & $\begin{array}{c}p_{1}^{i}(1 \leq i \leq k), \\
1, p_{2}, p_{2}^{\prime}\end{array}$ & $k+3$ \\
\hline $4 n, n>0$ & $\begin{array}{c}z^{n} p_{1}^{i}(0 \leq i \leq k-1), \\
z^{n} p_{2}, z^{n} p_{2}^{\prime}\end{array}$ & $k+2$ \\
\hline $4 n+1$ & $\begin{array}{r}u_{2} z^{n} p_{1}^{i}(0 \leq i \leq k-2), \\
u_{4} z^{n}, u_{4}^{\prime} z^{n}\end{array}$ & $k+1$ \\
\hline $4 n+2$ & $\begin{array}{r}v_{1} z^{n} p_{1}^{i}(0 \leq i \leq k-2), \\
v_{0} z^{n}, v_{0}^{\prime} z^{n}\end{array}$ & $k+1$ \\
\hline $4 n+3$ & $\begin{array}{c}w z^{n} p_{1}^{i}(0 \leq i \leq k-2), \\
w_{1} z^{n}, w_{1} z^{n} p_{2}^{\prime}, w_{1}^{\prime} z^{n}\end{array}$ & $k+2$ \\
\hline
\end{tabular}

This leads to (4.31).

Case 2: $p$ does not divide $3-2 k$ and divides $k$. Consider the following homogeneous elements in $\mathrm{HH}^{*}(R)$ :

$$
\begin{array}{lll}
\text { of degree 0: } & p_{1}, p_{2}, p_{2}^{\prime} \quad(\text { see (3.4) }) ; \\
\text { of degree 1: } & u_{2}, u_{4}, u_{4}^{\prime} \quad(\text { see (4.28) }) ; \\
\text { of degree 2: } & v_{0}, v_{0}^{\prime}, v_{1} \quad(\text { see (4.15) }) ; \\
\text { of degree 3: } & \widetilde{w}:=1, w_{1}:=x, w_{1}^{\prime}:=y ; \\
\text { of degree } 4: & z:=1 .
\end{array}
$$

Proposition 4.3.5. Assume that $p$ does not divide $3-2 k$ and divides $k$. Then, in the algebra $\mathrm{HH}^{*}(R)$, the elements of the set

$$
\mathcal{Y}_{6}=\left\{p_{1}, p_{2}, p_{2}^{\prime}, u_{2}, u_{4}, u_{4}^{\prime}, v_{0}, v_{0}^{\prime}, v_{1}, \widetilde{w}, w_{1}, w_{1}^{\prime}, z\right\}
$$

satisfy relations (4.17), (4.18), (4.19), and (4.30), and the relations

$$
\begin{gathered}
u_{4} v_{0}=\frac{1}{2} p_{1}^{k} \widetilde{w}, \quad u_{2} v_{1}=\frac{1}{2} p_{1}^{2} \widetilde{w}, \quad p_{2} \widetilde{w}=p_{2}^{\prime} \widetilde{w}=0 ; \\
u_{2} \widetilde{w}=u_{4} \widetilde{w}=u_{4}^{\prime} \widetilde{w}=v_{0} \widetilde{w}=v_{0}^{\prime} \widetilde{w}=0, \quad v_{1} \widetilde{w}=-2 u_{2} z ; \\
w_{1} \widetilde{w}=w_{1}^{\prime} \widetilde{w}=0 .
\end{gathered}
$$

Proof. The arguments are similar to those in the proof of Proposition 4.1.1. We observe additionally that the required translates of the elements of $\mathcal{Y}_{6}$ have been calculated earlier, and a large part of the relations mentioned above have already been established.

Now, the proof of part $3 \mathrm{~b}$ ) of Theorem 1.1 can be completed as in the previous cases. In particular, on the ring $K\left[\mathcal{X}_{6}\right]$ (with $\mathcal{X}_{6}$ as in (1.36) ), we use the lexicographic order such that

$$
v_{0}^{\prime}>v_{0}>v_{1}>u_{2}>u_{4}^{\prime}>u_{4}>\widetilde{w}>w_{1}^{\prime}>w_{1}>z>p_{1}>p_{2}>p_{2}^{\prime} .
$$


Then we introduce a list of elementary steps of reduction, obtained from the list (4.32) by deleting the steps that involve $w$ and adding the following steps to this list:

$$
u_{2} v_{1} \mapsto p_{1}^{2} \widetilde{w}, \quad v_{1} \widetilde{w} \mapsto u_{2} z, \quad \quad p^{k} \widetilde{w} \mapsto p_{2} w_{1}^{\prime} .
$$

After that, we show that the monomials having a normal form (with respect to the steps of reduction mentioned above) are as follows:

\begin{tabular}{|c|c|c|}
\hline Degree & Normal forms & Number \\
\hline \hline $4 n$ & $\begin{array}{c}z^{n} p_{1}^{i}(0 \leq i \leq k), \\
z^{n} p_{2}, z^{n} p_{2}^{\prime}\end{array}$ & $k+3$ \\
\hline $4 n+1$ & $\begin{array}{c}u_{2} z^{n} p_{1}^{i}(0 \leq i \leq k-2), \\
u_{4} z^{n}, u_{4}^{\prime} z^{n}\end{array}$ & $k+1$ \\
\hline $4 n+2$ & $\begin{array}{c}v_{1} z^{n} p_{1}^{i}(0 \leq i \leq k-2), \\
v_{0} z^{n}, v_{0}^{\prime} z^{n}\end{array}$ & $k+1$ \\
\hline $4 n+3$ & $\begin{array}{c}\widetilde{w} z^{n} p_{1}^{i}(0 \leq i \leq k-1), \\
w_{1} z^{n}, w_{1} z^{n} p_{2}^{\prime}, w_{1}^{\prime} z^{n}\end{array}$ & $k+3$ \\
\hline
\end{tabular}

Case 3: $p$ divides $3-2 k$; it is clear that $p$ does not divide $k$. Consider the following homogeneous elements in $\mathrm{HH}^{*}(R)$ :

$$
\begin{array}{lll}
\text { of degree 0: } & p_{1}, p_{2}, p_{2}^{\prime} \quad(\text { see (3.4) }) ; \\
\text { of degree 1: } & \widetilde{u}_{3}:=(x, y) ; \\
\text { of degree 2: } & v_{0}, v_{0}^{\prime}, v_{1} \quad(\text { see (4.15) }) ; \\
\text { of degree 4: } & z:=1 .
\end{array}
$$

Proposition 4.3.6. Assume that $p$ divides $3-2 k$. Then, in the algebra $\operatorname{HH}^{*}(R)$, the elements of the set

$$
\mathcal{Y}_{7}=\left\{p_{1}, p_{2}, p_{2}^{\prime}, \widetilde{u}_{3}, v_{0}, v_{0}^{\prime}, v_{1}, z\right\}
$$

satisfy relations (4.17), (4.18), and (4.21), and also the relations

$$
p_{2} v_{0}^{\prime}=p_{2}^{\prime} v_{0}, p_{1}^{k} \tilde{u}_{3}=p_{1}^{k} z=0 .
$$

The proof is similar to that of Proposition 4.1.1. We need to use the translates of the element $\widetilde{u}_{3}$ that are presented in the following lemma.

Lemma 4.3.7. For the role of the translates (up to first order inclusive) of the element $\widetilde{u}_{3}$, we can take the following maps:

$$
\mathrm{T}^{0}\left(\widetilde{u}_{3}\right)=(x \otimes 1, y \otimes 1)
$$$$
\mathrm{T}^{1}\left(\widetilde{u}_{3}\right)=
$$

$$
\left(\begin{array}{cc}
-x \otimes 1+\sum_{i=1}^{k-2}(2 i+1) y(x y)^{i} \otimes y(x y)^{k-2-i} & \sum_{i=1}^{k-1} 2 i(x y)^{i} \otimes(y x)^{k-1-i} \\
\sum_{i=1}^{k-1} 2 i(y x)^{i} \otimes(x y)^{k-1-i} & -y \otimes 1+\sum_{i=0}^{k-2}(2 i+1) x(y x)^{i} \otimes x(y x)^{k-2-i}
\end{array}\right) .
$$

Now, the proof of part $3 \mathrm{c}$ ) of Theorem 1.1 can be completed as in the preceding cases. On the ring $K\left[\mathcal{X}_{7}\right]$ (with $\mathcal{X}_{7}$ as in (1.37) ), we use the lexicographic order such that

$$
v_{0}^{\prime}>v_{0}>v_{1}>\widetilde{u}_{3}>z>p_{1}>p_{2}>p_{2}^{\prime} .
$$


Then we introduce the following list of elementary steps of reduction:

$$
\begin{aligned}
v_{0}^{2} \mapsto p_{2}^{\prime} z, & \left(v_{0}^{\prime}\right)^{2} \mapsto p_{2} z, \\
v_{1}^{2} \mapsto p_{1}^{2} z, & p_{2} v_{0}^{\prime} \mapsto p_{2}^{\prime} v_{0},
\end{aligned}
$$

and show that all monomials having a normal form (with respect to the steps of reduction

\begin{tabular}{|c|c|c|}
\hline Degree & Normal forms & Number \\
\hline 0 & $\begin{array}{c}p_{1}^{i}(1 \leq i \leq k) \\
1, p_{2}, p_{2}^{\prime}\end{array}$ & $k+3$ \\
\hline $4 n, n>0$ & $\begin{array}{c}z^{n} p_{1}^{i}(0 \leq i \leq k-1), \\
z^{n} p_{2}, z^{n} p_{2}^{\prime}\end{array}$ & $k+2$ \\
\hline $4 n+1$ & $\begin{array}{c}\widetilde{u}_{3} z^{n} p_{1}^{i}(0 \leq i \leq k-1) \\
\widetilde{u}_{3} z^{n} p_{2}, \widetilde{u}_{3} z^{n} p_{2}^{\prime}\end{array}$ & $k+2$ \\
\hline $4 n+2$ & $\begin{array}{c}v_{1} z^{n} p_{1}^{i}(0 \leq i \leq k-2) \\
v_{0} z^{n}, v_{0} z^{n} p_{2}^{\prime}, v_{0}^{\prime} z^{n}\end{array}$ & $k+2$ \\
\hline $4 n+3$ & $\begin{array}{l}v_{1} \widetilde{u}_{3} z^{n} p_{1}^{i}(0 \leq i \leq k-2), \\
v_{0} \widetilde{u}_{3} z^{n}, v_{0} \widetilde{u}_{3} z^{n} p_{2}^{\prime}, v_{0}^{\prime} \widetilde{u}_{3} z^{n}\end{array}$ & $k+2$ \\
\hline
\end{tabular}
mentioned above) are on the following list:

This completes the proof of Theorem 1.1

\section{§5. Appendix. The Yoneda Algebra}

Here we give (without a detailed proof) the description of the Yoneda algebra for the algebras $R_{k}(k \geq 2)$.

Let $R=R_{k}$, and let $S$ be a unique simple left $R$-module. The Yoneda algebra $\mathcal{Y}(R)$ of the algebra $R$ is a direct sum $\sum_{m \geq 0} \operatorname{Ext}_{R}^{m}(S, S)$ with multiplication given by the Yoneda product.

Proposition 5.1. The module $S$ is $\Omega$-periodic with period 4, and its minimal projective resolution is of the form

$$
0 \longleftarrow S \longleftarrow R \stackrel{(x, y)}{\longleftarrow} R^{2} \stackrel{\left(\begin{array}{cc}
x \\
-(y x)^{k-1} & -(x y)^{k-1} \\
y
\end{array}\right)}{\longleftarrow} R^{2} \stackrel{\left(\begin{array}{l}
x \\
y
\end{array}\right)}{\longleftarrow} R \stackrel{(x y)^{k}}{\longleftarrow} R \stackrel{(x, y)}{\longleftarrow} R^{2} \longleftarrow \ldots
$$

The proof consists in a direct verification of the fact that the sequence presented above is exact. Recall that the entries of the matrices of the differentials are endomorphisms of the left $R$-module $R$ that are induced by multiplication on the right by the corresponding elements of the algebra $R$.

Now, using the techniques of the papers [24, 25], we can prove the following statement.

Theorem 5.2. Let $R=R_{k}$ with $k \geq 2$. Then, as a graded algebra, the Yoneda algebra $\mathcal{Y}(R)$ is isomorphic to the algebra

$$
K[\xi, \zeta, \eta] /\left(\xi \zeta, \xi^{3}-\zeta^{3}, \xi^{4}, \zeta^{4}\right),
$$

where $\operatorname{deg} \xi=\operatorname{deg} \zeta=1, \operatorname{deg} \eta=4$.

Remark 5.3. Let $G$ be the generalized quaternion group, and let $K=\mathbb{F}_{2}$. The corresponding cohomology ring $\mathrm{H}^{*}(G, K)=\mathcal{Y}(K G)$ was calculated earlier in [26]. 


\section{REFERENCES}

[1] S. Eilenberg and S. MacLane, Cohomology theory in abstract groups. I, Ann. of Math. (2) 48 (1947), 51-78. MR0019092 (8:367f)

[2] H. Cartan and S. Eilenberg, Homological algebra, Princeton Univ. Press, Princeton, NJ, 1956. MR0077480 (17:1040e)

[3] M. Gerstenhaber, The cohomology structure of an associative ring, Ann. of Math. (2) 78 (1963), 267-288. MR0161898 (28:5102)

[4] M. Gerstenhaber and S. D. Schack, Algebraic cohomology and deformation theory, Deformation Theory of Algebras and Structures and Applications (Il Ciocco, 1986) (M. Hazewinkel, M. Gerstenhaber, eds.), NATO Adv. Sci. Inst. Ser. C Math. Phys. Sci., vol. 247, Kluwer Acad. Publ., Dordrecht, 1988, pp. 11-264. MR0981619 (90c:16016)

[5] Th. Holm, The Hochschild cohomology ring of a modular group algebra: The commutative case, Comm. Algebra 24 (1996), 1957-1969. MR.1386022 (97c:13012)

[6] C. Cibils and A. Solotar, Hochschild cohomology algebra of abelian groups, Arch. Math. (Basel) 68 (1997), 17-21. MR.1421841 (97k:13018)

[7] S. F. Siegel and S. J. Witherspoon, The Hochschild cohomology ring of a group algebra, Proc. London Math. Soc. (3) 79 (1999), 131-157. MR.1687539 (2000b:16016)

[8] K. Erdmann and Th. Holm, Twisted bimodules and Hochschild cohomology for self-injective algebras of class $A_{n}$, Forum Math. 11 (1999), 177-201. MR.1680594 (2001c:16018)

[9] K. Erdmann, Th. Holm, and N. Snashall, Twisted bimodules and Hochschild cohomology for selfinjective algebras of class $A_{n}$. II, Algebr. Represent. Theory 5 (2002), 457-482. MR1935856 (2004a:16013)

[10] Th. Holm, Hochschild cohomology of tame blocks, J. Algebra 271 (2004), 798-826. MR2025551 (2005c:20019)

[11] A. I. Generalov, Hochschild cohomology of algebras of dihedral type. I. The family $D(3 K)$ in characteristic 2, Algebra i Analiz 16 (2004), no. 6, 53-122; English transl., St. Petersburg Math. J. 16 (2005), no. 6, 961-1012. MR2117449 (2005i:16013)

[12] K. Erdmann, Blocks of tame representation type and related algebras, Lecture Notes in Math., vol. 1428, Springer-Verlag, Berlin, 1990. MR.1064107 (91c:20016)

[13] Th. Holm, Derived equivalence classification of algebras of dihedral, semidihedral, and quaternion type, J. Algebra 211 (1999), 159-205. MR1656577 (2000a:16019)

[14] A. I. Generalov, Cohomology of algebras of semidihedral type. I, Algebra i Analiz 13 (2001), no. 4, 54-85; English transl., St. Petersburg Math. J. 13 (2002), no. 4, 549-573. MR.1865495|(2002h:16020)

[15] A. I. Generalov and E. A. Osiyuk, Cohomology of algebras of dihedral type, III. The D(2A) series, Zap. Nauchn. Sem. S.-Peterburg. Otdel. Mat. Inst. Steklov. (POMI) 289 (2002), 113-133; English transl., J. Math. Sci. (N.Y.) 124 (2004), no. 1, 4741-4753. MR.1949737 (2003k:16015)

[16] A. I. Generalov, Cohomology of algebras of dihedral type, IV: $D(2 B)$ series, Zap. Nauchn. Sem. S.-Peterburg. Otdel. Mat. Inst. Steklov. (POMI) 289 (2002), 76-89; English transl., J. Math. Sci. (N.Y.) 124 (2004), no. 1, 4719-4726. MR.1949735 (2003k:16016)

[17] M. A. Antipov and A. I. Generalov, Cohomology of algebras of semidihedral type. II, Zap. Nauchn. Sem. S.-Peterburg. Otdel. Mat. Inst. Steklov. (POMI) 289 (2002), 9-36; English transl., J. Math. Sci. (N.Y.) 124 (2004), no. 1, 4681-4697. MR1949731 (2003k:16022)

[18] A. I. Generalov, Cohomology of algebras of semidihedral type, III. The series $S D(3 K)$, Zap. Nauchn. Sem. S.-Peterburg. Otdel. Mat. Inst. Steklov. (POMI) 305 (2003), 84-100; English transl., J. Math. Sci. (N.Y.) 130 (2005), no. 3, 4689-4698. MR2033615 (2004m:16016)

[19] _ Cohomology of algebras of semidihedral type. IV, Zap. Nauchn. Sem. S.-Peterburg. Otdel. Mat. Inst. Steklov. (POMI) 319 (2004), 81-116; English transl., J. Math. Sci. (N.Y.) 134 (2006), no. 6, 2489-2510. MR 2117855 (2005k:16019)

[20] E. L. Green and N. Snashall, Projective bimodule resolutions of an algebra and vanishing of the second Hochschild cohomology group, Forum Math. 16 (2004), 17-36. MR.2034541 (2005c:16011)

[21] M. J. Bardzell, The alternating syzygy behavior of monomial algebras, J. Algebra 188 (1997), 69-89. MR:1432347 (98a:16009)

[22] D. Happel, Hochschild cohomology of finite-dimensional algebras, Séminaire d'Algèbre Paul Dubreil et Marie-Paul Malliavin, 39ème Année (Paris, 1987/1988), Lecture Notes in Math., vol. 1404, Springer, Berlin, 1989, pp. 108-126. MR1035222 (91b:16012)

[23] A. I. Generalov and M. A. Kachalova, Bimodule resolution of the Möbius algebra, Zap. Nauchn. Sem. S.-Peterburg. Otdel. Mat. Inst. Steklov. (POMI) 321 (2005), 36-66. (Russian) MR2138411 (2006a:16014) 
[24] A. I. Generalov, Cohomology of algebras of dihedral type. I, Zap. Nauchn. Sem. S.-Peterburg. Otdel. Mat. Inst. Steklov. (POMI) 265 (1999), 139-162 (2000); English transl., J. Math. Sci. (N.Y.) 112 (2002), no. 3, 4318-4331. MR1757820 (2001b:20090)

[25] O. I. Balashov and A. I. Generalov, The Yoneda algebras for some class of dihedral algebras, Vestnik S.-Peterburg. Univ. Ser. 1 1999, vyp. 3, 3-10; English transl., Vestnik St. Petersburg Univ. Math. 32 (1999), no. 3, 1-8 (2000). MR1794988 (2001m:16020)

[26] J. Martino and S. Priddy, Classification of BG for groups with dihedral or quaternion Sylow 2subgroups, J. Pure Appl. Algebra 73 (1991), 13-21. MR1121628 (92f:55022)

Department of Mathematics and Mechanics, St. Petersburg State University, Universitetskiĭ Pr. 28, Stary ̌ Peterhof, St. Petersburg 198904, Russia

Received 19/SEP/2005

Translated by THE AUTHOR 